\title{
A JET BREAK IN THE X-RAY LIGHT CURVE OF SHORT GRB 111020A: IMPLICATIONS FOR ENERGETICS AND RATES
}

\author{
W. Fong ${ }^{1}$, E. Berger ${ }^{1}$, R. Margutti ${ }^{1}$, B. A. Zauderer ${ }^{1}$, E. Troja ${ }^{2}$, I. CZekala $^{1}$, R. Chornock ${ }^{1}$, \\ N. Gehrels ${ }^{2}$, T. Sakamoto ${ }^{2}$, D. B. Fox ${ }^{3}$, And P. Podsiadlowski ${ }^{4}$ \\ ${ }^{1}$ Harvard-Smithsonian Center for Astrophysics, 60 Garden Street, Cambridge, MA 02138, USA \\ ${ }^{2}$ NASA Goddard Space Flight Center, Greenbelt, MD 20771, USA \\ ${ }^{3}$ Department of Astronomy and Astrophysics, 525 Davey Laboratory, Pennsylvania State University, University Park, PA 16802, USA \\ ${ }^{4}$ Department of Astronomy, Oxford University, Oxford OX1 3RH, UK \\ Received 2012 April 23; accepted 2012 July 18; published 2012 August 27
}

\begin{abstract}
We present broadband observations of the afterglow and environment of the short GRB 111020A. An extensive X-ray light curve from Swift/XRT, XMM-Newton, and Chandra, spanning $\sim 100$ s to 10 days after the burst, reveals a significant break at $\delta t \approx 2$ days with pre- and post-break decline rates of $\alpha_{X, 1} \approx-0.78$ and $\alpha_{X, 2} \lesssim-1.7$, respectively. Interpreted as a jet break, we infer a collimated outflow with an opening angle of $\theta_{j} \approx 3^{\circ}-8^{\circ}$. The resulting beaming-corrected $\gamma$-ray $\left(10-1000 \mathrm{keV}\right.$ band) and blast-wave kinetic energies are (2-3) $\times 10^{48} \mathrm{erg}$ and $(0.3-2) \times 10^{49} \mathrm{erg}$, respectively, with the range depending on the unknown redshift of the burst. We report a radio afterglow limit of $<39 \mu \mathrm{Jy}(3 \sigma)$ from Expanded Very Large Array observations that, along with our finding that $v_{c}<v_{X}$, constrains the circumburst density to $n_{0} \sim 0.01-0.1 \mathrm{~cm}^{-3}$. Optical observations provide an afterglow limit of $i \gtrsim 24.4 \mathrm{mag}$ at $18 \mathrm{hr}$ after the burst and reveal a potential host galaxy with $i \approx 24.3 \mathrm{mag}$. The subarcsecond localization from Chandra provides a precise offset of $0.80 \pm 0$ '. $11(1 \sigma)$ from this galaxy corresponding to an offset of 5-7 kpc for $z=0.5-1.5$. We find a high excess neutral hydrogen column density of $(7.5 \pm 2.0) \times 10^{21} \mathrm{~cm}^{-2}$ $(z=0)$. Our observations demonstrate that a growing fraction of short gamma-ray bursts (GRBs) are collimated, which may lead to a true event rate of $\gtrsim 100-1000 \mathrm{Gpc}^{-3} \mathrm{yr}^{-1}$, in good agreement with the NS-NS merger rate of $\approx 200-3000 \mathrm{Gpc}^{-3} \mathrm{yr}^{-1}$. This consistency is promising for coincident short GRB-gravitational wave searches in the forthcoming era of Advanced LIGO/VIRGO.
\end{abstract}

Key words: gamma-ray burst: general - gamma-ray burst: individual (111020A)

Online-only material: color figures

\section{INTRODUCTION}

Observations of the temporal and spectral evolution of shortduration gamma-ray burst (GRB, $T_{90}<2 \mathrm{~s}$; Kouveliotou et al. 1993) afterglows are crucial to our understanding of the basic properties of these events: their energetics, parsec-scale environments, and geometries. From observations over the past 7 years, we now know that short GRBs have isotropic-equivalent energies of $\sim 10^{50}-10^{52} \mathrm{erg}$ (Berger 2007) and circumburst densities of $\sim 10^{-6}$ to $1 \mathrm{~cm}^{-3}$ (Soderberg et al. 2006; Panaitescu 2006; Stratta et al. 2007; Perley et al. 2009b; Berger 2010; Fong et al. 2011); however, these ranges are based on only a handful of events. The geometry, or degree of collimation, is the least constrained property but is of particular interest because it directly affects the true energy scale and event rates. These parameters aid our understanding of the explosion physics, the nature of the progenitors, and the potential detectability of short GRBs as gravitational wave sources. In particular, knowledge of the true energy scale may constrain the mechanism of energy extraction from the central engine and the ejecta composition: $v \bar{v}$ annihilation powering a baryonic jet (Jaroszynski 1993; Mochkovitch et al. 1993) or magnetohydrodynamic (MHD) processes in a magnetically dominated outflow (Blandford \& Znajek 1977; Rosswog et al. 2003). Significant improvement on the short GRB observed rate of $\gtrsim 10 \mathrm{Gpc}^{-3} \mathrm{yr}^{-1}$ (Nakar \& Granot 2007) will have a critical impact on estimates for coincident short GRB-gravitational wave detections in the era of Advanced LIGO/VIRGO (Abadie et al. 2010).

The opening angles $\left(\theta_{j}\right)$ of GRBs can be inferred from temporal breaks in the afterglow light curves ("jet breaks"), which occur at the time, $t_{j}$, when the Lorentz factor of the outflow is $\Gamma\left(t_{j}\right) \approx 1 / \theta_{j}$; a later break corresponds to a wider opening angle (Sari et al. 1999; Rhoads 1999). Jet breaks in the light curves of long-duration GRBs have led to an opening angle distribution with a range of $\sim 2^{\circ}-20^{\circ}$ and a median of $7^{\circ}$, leading to beaming-corrected energies of $E_{\gamma}=\left[1-\cos \left(\theta_{j}\right)\right] E_{\gamma, \text { iso }} \sim$ $10^{50}-10^{51} \mathrm{erg}$ (Bloom et al. 2003; Frail et al. 2001; Friedman \& Bloom 2005; Kocevski \& Butler 2008; Racusin et al. 2009). For short GRBs, there is mounting theoretical (Eichler et al. 1989; Narayan et al. 1992) and observational (Fong et al. 2010; Berger 2010; Church et al. 2011) evidence that the progenitors are NS-NS/NS-BH mergers, and numerous simulations of postmerger black hole $(\mathrm{BH})$ accretion have predicted collimated outflows with $\theta_{j} \sim 5^{\circ}-20^{\circ}$ (Popham et al. 1999; Aloy et al. 2005; Rosswog 2005; Rezzolla et al. 2011) up to several tens of degrees (Ruffert \& Janka 1999b; Popham et al. 1999; Rezzolla et al. 2011).

However, the detection of jet breaks in the afterglow light curves of short GRBs has proved to be challenging. They can in principle be measured from optical or radio observations, but there are several caveats that make this particularly difficult for short GRBs. First, the brightness of optical and radio afterglows is sensitive to the circumburst densities, which are typically low, $\sim 10^{-2} \mathrm{~cm}^{-3}$ (Soderberg et al. 2006). Indeed, of nearly 70 short bursts detected by Swift, only 2 radio afterglows have been detected over the past 7 years (Berger et al. 2005; Soderberg et al. 2006; Chandra \& Frail 2011). Similarly, only 30\% of Swift bursts have detected optical afterglows, with a typical brightness at $\lesssim 1$ day of $\approx 23 \mathrm{mag}$ (Berger 2010; Fong et al. 2011), making long-term temporal monitoring nearly impossible with 
ground-based facilities. Second, in the optical band there can be significant contamination from the host galaxies, which are generally brighter than the afterglows at $\gtrsim 1$ day (Berger 2010).

On the other hand, the X-ray afterglow brightness is independent of the circumburst density (as long as the density is $\gtrsim 10^{-5} \mathrm{~cm}^{-3}$ and hence $v_{c}>v_{X}$; Granot \& Sari 2002), and host contamination is not an issue. In addition, the well-sampled Swift/XRT light curves from $\sim 1$ minute to $\sim 1$ day provide an unambiguous baseline against which we can measure a subsequent break. Therefore, it is no surprise that the X-rays enabled the discovery of the first jet break in a short GRB. The X-ray afterglow light curve of GRB 051221A exhibited a break at $\approx 5$ days, leading to $\theta_{j} \approx 7^{\circ}$ (Soderberg et al. 2006; Burrows et al. 2006). Similarly, Chandra observations of GRB 050724A out to 22 days placed a meaningful lower limit of $\theta_{j} \gtrsim 25^{\circ}$ (Grupe et al. 2006), consistent with a spherical explosion. Temporal breaks on timescales of $\lesssim$ few hours were observed in the XRT light curves of GRBs 061201 (Stratta et al. 2007) and $090510^{5}$ (De Pasquale et al. 2010). If these are interpreted as jet breaks, they would lead to $\theta_{j} \sim 1^{\circ}$; however, they also match the timescale and behavior of early breaks in long GRBs, which are not due to collimation (Nousek et al. 2006; Zhang et al. 2006; Liang et al. 2007). Finally, there is tentative evidence for beaming in the light curves of GRBs 050709 (Fox et al. 2005) and 061210 (Berger 2007); however, these are based on sparsely sampled light curves without a definitive break (e.g., Watson et al. 2006). No other jet breaks in the light curves of unambiguous short GRBs have been reported to date, ${ }^{7}$ and the lack of jet breaks in Swift/XRT observations out to $\sim 1-2$ days can provide only weak lower bounds of $\theta_{j} \gtrsim 2^{\circ}-6^{\circ}$ (revised from Coward et al. 2012 with more realistic density values; see Section 4.2).

Against this backdrop, we present here the discovery of a break in the X-ray light curve of GRB $111020 \mathrm{~A}$ at $\delta t \approx 2$ days, best explained as a jet break. We also present contemporaneous radio and optical limits on the afterglow, allowing a characterization of the broadband synchrotron spectrum and constraints on the energy and density. In addition, we report the discovery of a putative host galaxy. A comparison of our X-ray and optical data may require an appreciable amount of extinction and the highest intrinsic neutral hydrogen column density for a short GRB to date. Our results have implications for the opening angle distribution and therefore the observed short GRB rate and true energy release.

Unless otherwise noted, all magnitudes in this paper are in the $\mathrm{AB}$ system and are corrected for Galactic extinction in the direction of the burst using $E(B-V)=0.432 \mathrm{mag}$ (Schlegel et al. 1998; Schlafly \& Finkbeiner 2011). We employ a standard $\Lambda$ CDM cosmology with $\Omega_{M}=0.27, \Omega_{\Lambda}=0.73$, and $H_{0}=71 \mathrm{~km} \mathrm{~s}^{-1} \mathrm{Mpc}^{-1}$.

\section{OBSERVATIONS OF GRB 111020A}

\subsection{Swift Observations}

GRB 111020A was detected on 2011 October 20 at 06:33:49.0 UT by the Burst Alert Telescope (BAT) on board

\footnotetext{
5 GRB 090510 also exhibits a post-jet-break-like decay in its optical light curve (Nicuesa Guelbenzu et al. 2012).

6 Please note that Berger (2007) erroneously refers to GRB 061006.

7 A jet break was reported in the light curve of GRB 090426A (Nicuesa Guelbenzu et al. 2011); however, the characteristics of its environment and prompt emission are more similar to those of long GRBs (Levesque et al. 2010; Xin et al. 2011).
}

the Swift satellite (Gehrels et al. 2004; Sakamoto et al. 2011c). BAT located the burst at a ground-calculated position of R.A. = $19^{\mathrm{h}} 08^{\mathrm{m}} 06^{\mathrm{s}} .9$ and $\mathrm{decl} .=-38^{\circ} 01^{\prime} 50^{\prime \prime} \cdot 3(\mathrm{~J} 2000)$ with $2^{\prime} \cdot 1$ accuracy (90\% containment; Sakamoto et al. 2011b). The X-Ray Telescope (XRT) commenced observations of the location of the burst at $\delta t=72.8 \mathrm{~s}$ (where $\delta t$ is the time after the BAT trigger) and detected a fading X-ray source (Section 2.2). The UV-Optical Telescope (UVOT) began observations of the field at $\delta t=79 \mathrm{~s}$, but no corresponding UV or optical source was found within the XRT position. The $3 \sigma$ limit in the white filter, which transmits over $\lambda=1600-8000 \AA$ (Poole et al. 2008), is $20.3 \mathrm{mag}$ (not corrected for Galactic extinction; Oates \& Sakamoto 2011).

The gamma-ray emission consists of a single pulse with a duration of $T_{90}=0.40 \pm 0.09 \mathrm{~s}$ in the $15-350 \mathrm{keV}$ band, classifying GRB 111020A as a short burst (Sakamoto et al. 2011b). The spectrum is best fit with a single power law with index $1.37 \pm 0.26$ and a fluence of $f_{\gamma}=(6.5 \pm 1.0) \times$ $10^{-8} \mathrm{erg} \mathrm{cm}^{-2}(15-150 \mathrm{keV})$. Spectral lag analysis is not conclusive, and there is no clear evidence for extended emission (Sakamoto et al. 2011a).

\subsection{X-Ray Observations}

The XRT promptly located a fading, uncataloged X-ray source (Evans et al. 2007, 2009; Sakamoto et al. 2011c) with a UVOT-enhanced position of R.A. $=19^{\mathrm{h}} 08^{\mathrm{m}} 12^{\mathrm{s}} .53$ and decl. $=$ $-38^{\circ} 00^{\prime} 43^{\prime \prime} .8$ (J2000) and an uncertainty of 1".6 (Osborne et al. 2011). XRT observations of the field continued until the source faded below the detection threshold at $\delta t \approx 3.5$ days.

We also observed the field of GRB 111020A with the European Photon Imaging Camera (EPIC-PN) on board the $X$-ray Multi-Mirror Mission (XMM-Newton) starting at $\delta t=$ 0.65 days. With $13.5 \mathrm{ks}$ of on-source observations, we clearly detect a source in the energy range $0.5-10 \mathrm{keV}$, consistent with the Swift-XRT position. In addition, we obtained two sets of $20 \mathrm{ks}$ observations with the Advanced CCD Imaging Spectrometer (ACIS-S, 0.3-10 keV) on board the Chandra $X$-Ray Observatory at $\delta t=2.9$ and 10.1 days to refine the astrometry and monitor the light-curve evolution. We detect the X-ray afterglow in the first Chandra observation but do not detect any source at the same location in the second epoch.

\subsubsection{Data Analysis and Spectral Fitting}

We analyze the XRT data using the latest version of the HEASOFT package (ver. 6.11) and relevant calibration files. We apply standard filtering and screening criteria and generate a count rate light curve following the prescriptions from Margutti et al. (2010, 2012). Our re-binning scheme ensures a minimum signal-to-noise ratio of $\mathrm{S} / \mathrm{N}=4$ for each temporal bin. We analyze the $X M M$ data using standard routines in the Scientific Analysis System (SAS) ver. 11. We remove the first $5 \mathrm{ks}$ of data due to high background contamination, giving a total exposure time of $13.5 \mathrm{ks}$. We extract count rates from a $20^{\prime \prime}$ radius aperture, and the background is calculated using $20^{\prime \prime}$ radius source-free regions on the same chip. We use the CIAO data reduction package for the Chandra data. For the first epoch, we use a 2 '.5 radius source aperture centered on the Chandra position and a background annulus with inner and outer radii of $10^{\prime \prime}$ and $35^{\prime \prime}$, respectively, giving a source detection significance of $\sim 30 \sigma$. For the second epoch, we extract 1 count in a $2^{\prime \prime} .5$ aperture at the location of the source, consistent with the average background level calculated from source-free regions on the 
Table 1

GRB 111020A X-Ray Spectral Fit Parameters

\begin{tabular}{lcccrr}
\hline \hline Telescope & Detector & $\begin{array}{c}\delta t \\
(\mathrm{ks})\end{array}$ & $\begin{array}{c}N_{\mathrm{H}, \mathrm{int}}^{\mathrm{a}, \mathrm{b}} \\
\left(10^{22} \mathrm{~cm}^{-2}\right)\end{array}$ & $\Gamma^{\mathrm{a}, \mathrm{b}}$ & $C$-stat ${ }_{v} / \mathrm{dof}^{-}$ \\
\hline Swift & XRT & $0.08-60$ & $1.0 \pm 0.3$ & $2.2 \pm 0.5$ & $0.86 / 188$ \\
XMM & EPIC-PN & $61.4-76.8$ & $0.65_{-0.23}^{+0.21}$ & $2.0 \pm 0.4$ & $1.0 / 256$ \\
Chandra & ACIS-S & $250.5-268.5$ & $0.4_{-0.4}^{+2.3}$ & $1.1_{-0.8}^{+2.7}$ & $0.32 / 661$ \\
Swift+XMM & XRT+EPIC-PN & see above & $0.75_{-0.18}^{0.20}$ & $2.0 \pm 0.3$ & $0.94 / 446$ \\
\hline
\end{tabular}

Notes.

${ }^{\text {a }}$ These values assume a Galactic column density of $N_{\mathrm{H} \text {,gal }}=6.9 \times 10^{20} \mathrm{~cm}^{-2}$ (Kalberla et al. 2005), using an XSPEC model of tbabs $\times$ ztbabs $\times$ pow at $z=0$.

${ }^{\mathrm{b}}$ Uncertainties correspond to a $90 \%$ confidence level.

Table 2

GRB 111020A X-Ray Observations

\begin{tabular}{|c|c|c|}
\hline $\begin{array}{l}\delta t \\
(\mathrm{~s})\end{array}$ & $\begin{array}{c}\text { Time Bin Duration } \\
\text { (s) }\end{array}$ & $\begin{array}{c}\text { Unabs. Flux }(0.3-10 \mathrm{keV}) \\
\quad\left(\mathrm{erg} \mathrm{cm}^{-2} \mathrm{~s}^{-1}\right)\end{array}$ \\
\hline \multicolumn{3}{|c|}{ Swift/XRT } \\
\hline $6.18 \times 10^{1 \mathrm{a}}$ & $7.44 \times 10^{0}$ & $(2.38 \pm 0.79) \times 10^{-10}$ \\
\hline $1.35 \times 10^{2}$ & $4.64 \times 10^{1}$ & $(3.80 \pm 1.03) \times 10^{-11}$ \\
\hline $2.66 \times 10^{2}$ & $1.71 \times 10^{2}$ & $(1.64 \pm 0.42) \times 10^{-11}$ \\
\hline $4.15 \times 10^{2}$ & $1.26 \times 10^{2}$ & $(2.43 \pm 0.64) \times 10^{-11}$ \\
\hline $5.96 \times 10^{2}$ & $2.36 \times 10^{2}$ & $(1.03 \pm 0.26) \times 10^{-11}$ \\
\hline $7.97 \times 10^{2}$ & $1.66 \times 10^{2}$ & $(1.83 \pm 0.49) \times 10^{-11}$ \\
\hline $1.14 \times 10^{3}$ & $5.20 \times 10^{2}$ & $(8.85 \pm 1.72) \times 10^{-12}$ \\
\hline $5.94 \times 10^{3}$ & $2.46 \times 10^{3}$ & $(1.65 \pm 0.33) \times 10^{-12}$ \\
\hline $1.17 \times 10^{4}$ & $2.46 \times 10^{3}$ & $(1.07 \pm 0.27) \times 10^{-12}$ \\
\hline $1.94 \times 10^{4}$ & $6.38 \times 10^{3}$ & $(9.19 \pm 2.28) \times 10^{-13}$ \\
\hline $2.58 \times 10^{4}$ & $6.28 \times 10^{3}$ & $(1.19 \pm 0.32) \times 10^{-12}$ \\
\hline $3.19 \times 10^{4}$ & $5.90 \times 10^{3}$ & $(1.05 \pm 0.27) \times 10^{-12}$ \\
\hline $4.29 \times 10^{4}$ & $1.61 \times 10^{4}$ & $(8.36 \pm 2.41) \times 10^{-13}$ \\
\hline $1.26 \times 10^{5}$ & $1.51 \times 10^{5}$ & $(1.63 \pm 0.55) \times 10^{-13}$ \\
\hline $3.09 \times 10^{5}$ & $2.14 \times 10^{5}$ & $(1.11 \pm 0.42) \times 10^{-13}$ \\
\hline \multicolumn{3}{|c|}{$X M M /$ EPIC-PN } \\
\hline $6.91 \times 10^{4}$ & $1.35 \times 10^{4}$ & $(2.66 \pm 0.19) \times 10^{-13}$ \\
\hline \multicolumn{3}{|c|}{ Chandra/ACIS-S } \\
\hline $2.61 \times 10^{5}$ & $1.98 \times 10^{4}$ & $(5.96 \pm 0.89) \times 10^{-14}$ \\
\hline $8.84 \times 10^{5}$ & $1.98 \times 10^{4}$ & $<8.95 \times 10^{-15}$ \\
\hline
\end{tabular}

Notes. Upper limits are $3 \sigma$.

a These points were excluded from the broken power-law fit.

same chip. We therefore take the $3 \sigma$ background count rate as an upper limit on the X-ray afterglow.

To extract a spectrum from the X-ray data, we fit each of the data sets with an absorbed power-law model (tbabs $\times$ ztbabs $\times$ pow within the XSPEC routine) characterized by a photon index, $\Gamma$, and intrinsic neutral hydrogen absorption column, $N_{\mathrm{H}, \text { int }}$, in excess of the Galactic column density in the direction of the burst, $N_{\mathrm{H}, \mathrm{MW}}=6.9 \times 10^{20} \mathrm{~cm}^{-2}$ (typical uncertainty of $\sim 10 \%$; Kalberla et al. 2005; Wakker et al. 2011), using Cash statistics. For XRT, we utilize data in the time interval $0.08-60 \mathrm{ks}$ where there is no evidence for spectral evolution. We find an average best-fitting $\left(C\right.$-stat $_{v}=$ 0.86 for 188 dof) spectrum characterized by $\Gamma=2.2 \pm 0.5$ and $N_{\mathrm{H} \text {,int }}=(1.0 \pm 0.3) \times 10^{22} \mathrm{~cm}^{-2}$ at $z=0$ (Table 1). Uncertainties correspond to the $90 \%$ confidence level (CL). Our best-fit parameters are fully consistent with the automatic spectrum fit produced by Mangano \& Sakamoto (2011). The $X M M$ data are best modeled with a power law characterized by $\Gamma=2.0 \pm 0.4$ and $N_{\mathrm{H}, \text { int }}=(0.65 \pm 0.22) \times 10^{22} \mathrm{~cm}^{-2}$ $\left(C\right.$-stat $_{v}=1.0$ for 256 dof $)$, consistent with the XRT model

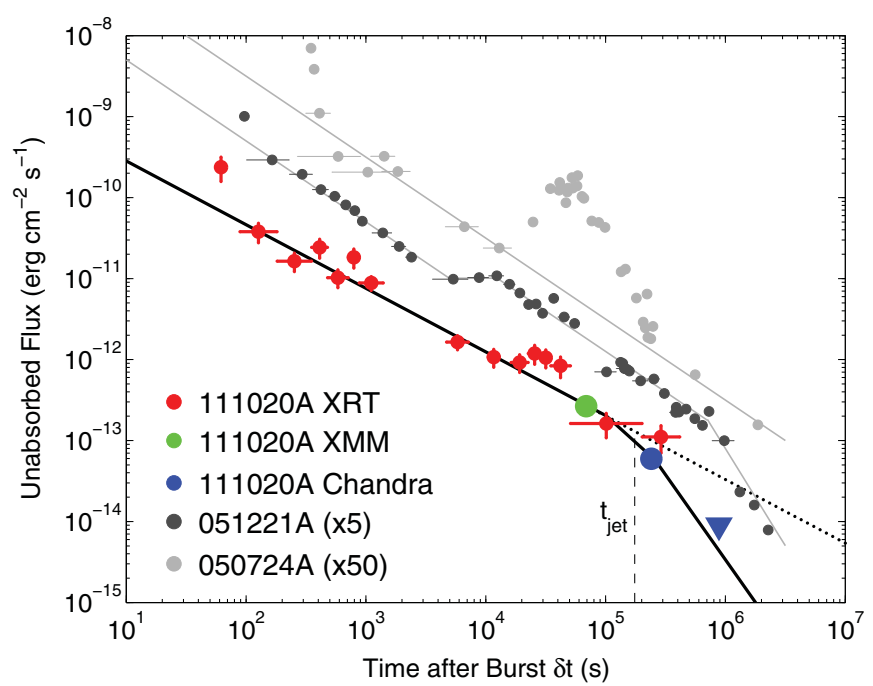

Figure 1. Unabsorbed X-ray flux light curve for GRB 111020A from SwiftXRT (red), XMM (green), and Chandra (blue). Flux errors are $1 \sigma$. The Chandra $3 \sigma$ upper limit is denoted by the blue triangle. The best-fit broken power-law model (black solid line) for GRB 111020A is characterized by $\alpha_{1}=-0.78$, $\alpha_{2}=-2.1$, and $t_{j}=2.0$ days. A single power-law model with $\alpha=-0.78$ (black dotted) violates the Chandra upper limit. Also plotted are X-ray light curves for short GRBs 051221A (dark gray circles; Soderberg et al. 2006; Burrows et al. 2006) and 050724 (light gray circles; Grupe et al. 2006). The data for GRBs 051221A and 050724 have been scaled for clarity. Gray lines trace the afterglow evolution with a break for GRB $051221 \mathrm{~A}$ at $\approx 5$ days and no break for GRB 050724 A to $\approx 22$ days.

(A color version of this figure is available in the online journal.)

parameters. We also fit the first epoch of Chandra data, and the resulting parameters are consistent with those from XRT and $X M M$, albeit with large error bars due to low count statistics (Table 1). Since we find no evidence for spectral evolution in the XRT data, we perform a joint XRT $+X M M$ spectral analysis to obtain the best constraints on $\Gamma$ and $N_{\mathrm{H} \text {,int. The resulting best-fit }}$ model has $\Gamma=2.0 \pm 0.3$ and $N_{\mathrm{H} \text {,int }}=(0.8 \pm 0.2) \times 10^{22} \mathrm{~cm}^{-2}$ $\left(90 \% \mathrm{CL}, C-\right.$ stat $_{v}=0.94$ for 446 dof). Although the redshift of the burst is unknown, we note that $\Gamma$ remains unchanged within its $1 \sigma$ value for $z \lesssim 3$, and we find evidence for intrinsic $N_{\mathrm{H} \text {,int }}$ in excess of the Galactic value at $6.5 \sigma$ confidence. The bestfitting spectral parameters for each of the three data sets and the joint fit are summarized in Table 1.

Applying these parameters to the individual XRT, XMM, and Chandra data sets, we calculate the count-rate-to-flux conversion factors and hence their absorbed and unabsorbed fluxes (Table 2). Applying these conversion factors results in the X-ray light curve shown in Figure 1. 

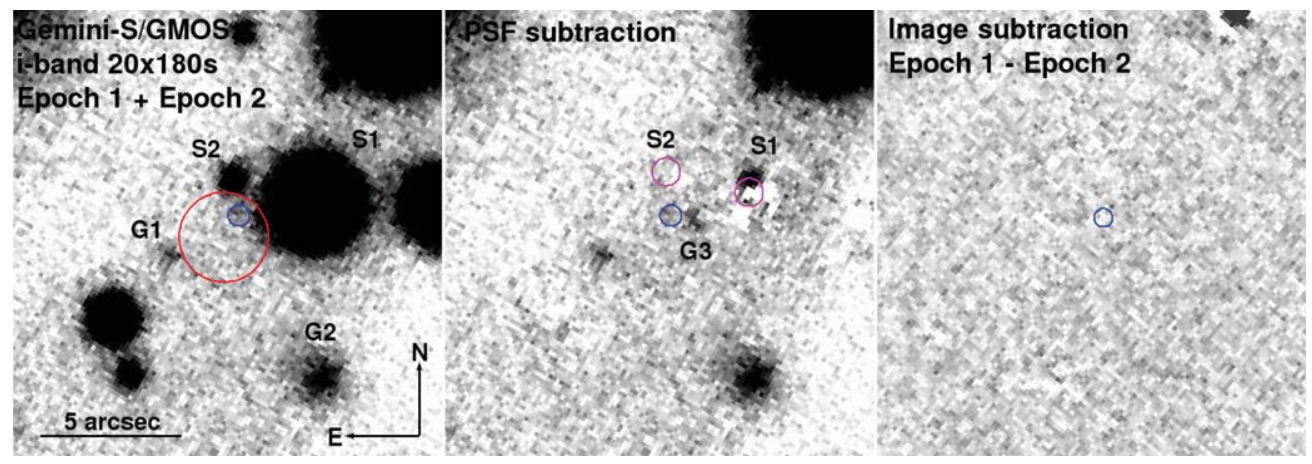

Figure 2. Optical $i$-band observations obtained with GMOS on Gemini-South. Left: combined stack of two nights of GMOS $i$-band data. Stars S1 and S2 are labeled, as well as galaxies G1 and G2. X-ray positions of GRB 111020A are denoted by the circles (red: Swift-XRT, 1'.6 radius, 90\% containment; blue: Chandra, 0'.33 radius, 90\% confidence). Center: PSF-subtracted image with the centroids of S1 and S2 (magenta circles). The subtraction reveals a third source, G3, with $i \approx 24.3$ mag. Right: digital image subtraction of the two epochs obtained at $17.7 \mathrm{hr}$ and 1.7 days, respectively, reveals no residuals in or around the Chandra position.

(A color version of this figure is available in the online journal.)

\subsubsection{Differential Astrometry}

In the absence of the detection of an optical afterglow (Section 2.3), we use our Chandra observations to refine the Swift/XRT position (1".6 uncertainty) to subarcsecond accuracy. We perform differential astrometry between our Chandra and Gemini Multi-Object Spectrograph (GMOS) observations (Section 2.3). To achieve the maximum signal-to-noise ratio, we combine both epochs of GMOS $i$-band observations and use SExtractor ${ }^{8}$ to determine the positions and centroid uncertainty of sources in the field. Performing an absolute astrometric tie to Two Micron All Sky Survey (2MASS) using $\sim 70$ common point sources, we find a resulting rms value of $\sigma_{\mathrm{GMOS}-2 \mathrm{MASS}}=0^{\prime \prime} 17$ ( $0^{\prime \prime} .12$ in each coordinate).

To refine the native Chandra astrometry and determine the location of the X-ray afterglow relative to the GMOS image, we perform differential astrometry. We use CIAO routines mergeall to combine the two Chandra epochs and wavdetect to obtain positions and $1 \sigma$ centroid uncertainties of X-ray sources in the field. We also use wavdetect to determine the Chandra position of the afterglow from the first epoch and find a $1 \sigma$ centroid statistical uncertainty $\sigma_{\mathrm{X}, \mathrm{ag}}=0^{\prime \prime} 08$. We calculate an astrometric tie based on two X-ray and optically bright common sources and find weighted mean offsets of $\delta$ R.A. $=-0^{\prime} .27 \pm 0$ '.06 and $\delta$ decl. $=+00^{\prime} 05 \pm 0$ 0.05, giving a tie uncertainty of $\sigma_{\mathrm{CXO}-\mathrm{GMOS}}=0^{\prime \prime} 08$. There are three additional common, but fainter, sources. An astrometric tie using all five sources gives weighted mean offsets and errors of $\delta$ R.A. $=-0{ }^{\prime} \cdot 29 \pm 0 . \prime 15$ and $\delta$ decl. $=+00^{\prime} 06 \pm 0$ ' 16 , fully consistent with our results from using the two bright sources alone. We therefore use the astrometric solution from the two bright sources only. Applying this solution, we obtain a Chandra X-ray afterglow position of R.A. $=19^{\mathrm{h}} 08^{\mathrm{m}} 12^{\mathrm{s}} .49$ and decl. $=$ $-38^{\circ} 00^{\prime} 42^{\prime \prime} .9$ (denoted by the blue circle in Figure 2) with a total $1 \sigma$ uncertainty of $0^{\prime} \cdot 20$, accounting for the 2MASS-GMOS astrometric tie, GMOS-Chandra tie, and afterglow positional uncertainty. This position is consistent with the XRT position and is offset by 0.94 from the XRT centroid.

\subsection{Optical Observations and Putative Host Galaxies}

We initiated $i$-band observations of GRB 111020A with the GMOS mounted on the Gemini-South $8 \mathrm{~m}$ telescope on 2011 October $21.01 \mathrm{UT}(\delta t=17.7 \mathrm{hr})$. The data were reduced using

\footnotetext{
http://sextractor.sourceforge.net/
}

the gemini/gmos package in IRAF. In a stack of $9 \times 180 \mathrm{~s}$ exposures in 0'.74 seeing and photometric conditions (Figure 2), we do not detect any sources within the enhanced XRT error circle or coincident with the Chandra position. However, the outskirts of the XRT position are partially contaminated by emission from a nearby $i=17.7 \mathrm{mag}$ star (S1) and a fainter star (S2) with $i=22.7 \mathrm{mag}$ (corrected for $A_{i}=0.73 \mathrm{mag}$, Figure 2). We detect two additional nearby sources: a faint galaxy (G1) located 2".8 away from the center of the Chandra position and a brighter galaxy (G2) with a 6."5 offset (Figure 2).

To search for a fading optical afterglow, we obtained a second, deeper set of $i$-band observations $(11 \times 180 \mathrm{~s})$ with GMOS at $\delta t=1.74$ days in $0{ }^{\prime} .67$ seeing. Digital image subtraction using the ISIS software package (Alard 2000) reveals no variation between the two epochs inside or near the X-ray afterglow error circles (Figure 2). To calculate the upper limit on the afterglow, we add several point sources of varying magnitudes in the range $i=24-26 \mathrm{mag}$ around the position in the first epoch using IRAF routine addstar. We perform photometry in the residual image in $2^{\prime \prime}$ apertures using the standard published $i$-band zero point for GMOS-S and place a $3 \sigma$ limit on the afterglow of $i \gtrsim 24.4 \mathrm{mag}\left(F_{\nu} \lesssim 0.63 \mu \mathrm{Jy}\right)$. We also perform photometry in a 1". 8 aperture for G1 and a 2".3 aperture for G2, giving magnitudes of $i=23.9 \pm 0.2 \mathrm{mag}$ and $i=21.9 \pm 0.1$, respectively (Table 3 ).

In addition, we obtained $r$-band observations $(3 \times 360 \mathrm{~s}$ in 0'62 seeing) with the Low-Resolution Survey Spectrograph 3 (LDSS3) mounted on the Magellan/Clay $6.5 \mathrm{~m}$ Telescope concurrent to the first epoch of GMOS observations $(\delta t=$ $17.7 \mathrm{hr}$ ). We obtained a second, deeper set of observations $\left(16 \times 150 \mathrm{~s}\right.$ in $\left.0{ }^{\prime \prime} 66 \mathrm{seeing}\right)$ at $\delta t \approx 180$ days, and digital image subtraction reveals no evidence for a fading source within the $\mathrm{X}$-ray positions to a $3 \sigma$ limit of $r \gtrsim 24.1 \mathrm{mag}$, where the zero point is determined from several standard stars at similar air mass. We easily detect G2, with $r=21.9 \pm 0.1 \mathrm{mag}$, but do not detect G1 to a $3 \sigma$ limit of $r \gtrsim 24.8$ (corrected for $A_{r}=$ $0.99 \mathrm{mag}$, Table 3). Finally, we obtained $z$-band observations with LDSS3 $\left(15 \times 180 \mathrm{~s}\right.$ in $0{ }^{\prime \prime} 60$ seeing $)$ at $\delta t \approx 180$ days. We detect $\mathrm{G} 1$ at $2.5 \sigma$ significance, $z=24.1 \pm 0.4$, and G2 with $z=21.7 \pm 0.1$ (corrected for $A_{z}=0.55 \mathrm{mag}$ ).

Since S1 and S2 contaminate the Chandra position, we subtract their contribution using point-spread function (PSF) subtraction on the individual observations and a combined stack of the two GMOS epochs. We use standard PSF-fitting routines in the IRAF daophot package. Modeling the PSF using four 
Table 3

GRB 111020A Optical Photometry

\begin{tabular}{|c|c|c|c|c|c|c|c|c|c|c|c|c|}
\hline $\begin{array}{l}\text { Date } \\
\text { (UT) }\end{array}$ & $\begin{array}{c}\delta t \\
\text { (days) }\end{array}$ & Telescope & Instrument & Filter & $\begin{array}{l}\text { Exposures } \\
\text { (s) }\end{array}$ & $\begin{array}{l}\theta_{\text {FWHM }} \\
(\operatorname{arcsec})\end{array}$ & $\begin{array}{l}\text { Afterglow }{ }^{a, b} \\
\text { (AB mag) }\end{array}$ & $\begin{array}{l}F_{v}^{\mathrm{a}, \mathrm{b}} \\
(\mu \mathrm{Jy})\end{array}$ & $\begin{array}{c}\mathrm{G} 1^{\mathrm{a}} \\
(\mathrm{AB} \mathrm{mag})\end{array}$ & $\begin{array}{c}\mathrm{G} 2^{\mathrm{a}} \\
(\mathrm{AB} \mathrm{mag})\end{array}$ & $\begin{array}{c}\mathrm{G}^{\mathrm{a}} \\
(\mathrm{AB} \mathrm{mag})\end{array}$ & $\begin{array}{c}A_{\lambda} \\
(\mathrm{AB} \mathrm{mag})\end{array}$ \\
\hline 2011 Oct 21.01 & 0.74 & Magellan/Clay & LDSS3 & $r$ & $3 \times 360$ & 0.62 & $>24.1$ & $<0.83$ & $>24.1$ & $22.00 \pm 0.08$ & $>24.1$ & 0.987 \\
\hline 2011 Oct 21.01 & 0.74 & Gemini-S & GMOS & $i$ & $9 \times 180$ & 0.74 & $>24.4$ & $<0.63$ & & & & 0.734 \\
\hline 2011 Oct 22.01 & 1.74 & Gemini-S & GMOS & $i$ & $11 \times 180$ & 0.67 & & & & & & 0.734 \\
\hline 2011 Oct $21+22$ & & Gemini-S & GMOS & $i$ & $20 \times 180$ & 0.72 & & & $23.89 \pm 0.17$ & $21.91 \pm 0.05$ & $24.27 \pm 0.16$ & 0.734 \\
\hline 2012 May 17.25 & 179.0 & Magellan/Clay & LDSS3 & $z$ & $15 \times 180$ & 0.60 & & & $24.05 \pm 0.41$ & $21.67 \pm 0.08$ & $>23.6$ & 0.546 \\
\hline 2012 May 17.30 & 179.1 & Magellan/Clay & LDSS3 & $r$ & $16 \times 180$ & 0.66 & & & $>24.8$ & $21.84 \pm 0.05$ & $>24.8$ & 0.987 \\
\hline
\end{tabular}

Notes.

a These values have been corrected for Galactic extinction $A_{\lambda}$ (Schlafly \& Finkbeiner 2011).

${ }^{\mathrm{b}}$ Limits are $3 \sigma$.

bright, unsaturated stars in the field out to a radius of $3^{\prime \prime}$ ( $\sim 4 \theta_{\text {FWHM }}$ ) from the center of each star, we subtract several stars in the field including S1 and S2. The clean subtraction of these stars indicates a model PSF representative of the PSF of the field. We uncover a faint, mildly extended source (G3) on the outskirts of S1 at coordinates R.A. $=19^{\mathrm{h}} 08^{\mathrm{m}} 12^{\mathrm{s}} .43$ and decl. $=-38^{\circ} 00^{\prime} 43^{\prime \prime} .07$ (J2000). This source, which lies 0'.80 from the center of the Chandra error circle, has a magnitude of $i=24.3 \pm 0.2$ and is a potential host of GRB 111020A (Section 3.1). G3 is not detected in the $r$ or $z$ filters to $3 \sigma$ limits of $r>24.8$ and $z>23.6$ (Table 3). Based on the limited color information, $r-i \gtrsim 0.5$ and $i-z \gtrsim-0.67$, we cannot rule out the possibility that this source is a faint star.

\subsection{Radio Observations and Possible Afterglow}

We observed the position of GRB 111020A with the Expanded Very Large Array ${ }^{9}$ (EVLA) beginning on 2011 October $20.95 \mathrm{UT}(\delta t=16.1 \mathrm{hr}$, Program 10C-145) at a mean frequency of $5.8 \mathrm{GHz}$ with a total on-source integration time of $65 \mathrm{~min}$ utes. We observed 3C 295 and J1937-1958 for bandpass/flux and gain calibration, respectively, and used standard procedures in the Astronomical Image Processing System (AIPS; Greisen 2003) for data calibration and analysis. With the new wideband capabilities of the EVLA (Perley et al. 2011), our data have an effective bandwidth of $\sim 1.5 \mathrm{GHz}$ after excising edge channels and data affected by radio frequency interference. The low declination of GRB 111020A and the compact D configuration of the array at the time of observation caused significant shadowing and required the removal of 7 out of 27 antennas (the north arm of the EVLA).

Taking into account the highly elongated beam $\left(33^{\prime \prime} \times 7^{\prime \prime}\right.$ with a position angle of $170^{\circ}$ ), we detect a $3.7 \sigma$ source with an integrated flux density of $48 \pm 13 \mu \mathrm{Jy}$ located at R.A. = $19^{\mathrm{h}} 08^{\mathrm{m}} 12^{\mathrm{s}} .40$, decl. $=-38^{\circ} 00^{\prime} 41^{\prime \prime} .2$ ( $\delta$ R.A. $=1^{\prime \prime} .1, \delta$ decl. $=$ 3". $6,1 \sigma$ uncertainty), consistent with the Chandra position. The position, peak flux, and integrated flux of the source are consistent regardless of our choice of weighting, or if we analyze the upper and lower sidebands separately. However, despite the statistical significance of the detection, we cannot completely rule out sidelobe contribution from nearby bright sources in the field due to the low declination of the burst. Therefore, we conservatively adopt a $3 \sigma$ upper limit of $39 \mu \mathrm{Jy}$ on the radio afterglow of GRB 111020A for our analysis. We note that if the source is indeed real, then upper limits inferred from the radio data can be treated as actual values.

\footnotetext{
9 Newly renamed the Karl G. Jansky Very Large Array.
}

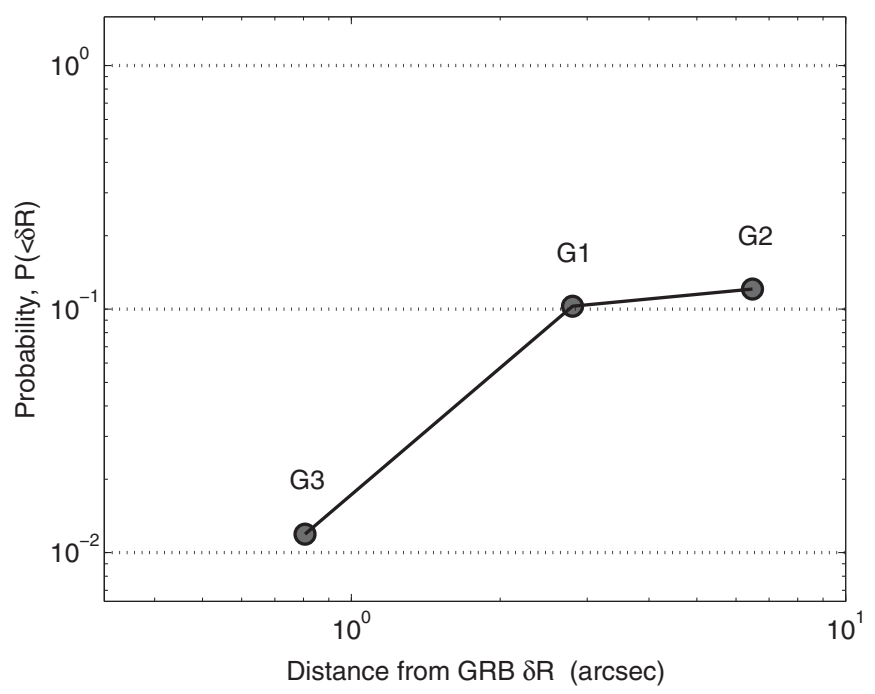

Figure 3. Probability of chance coincidence, $P(<\delta R)$, as a function of angular distance from the center of the Chandra afterglow position for the three host galaxy candidates of GRB $111020 \mathrm{~A}$. The galaxy G3 has the lowest probability of chance coincidence, $P(<\delta R)=0.01$, and is therefore the most probable host of GRB 111020A.

\section{RESULTS}

\subsection{Galaxy Probabilities of Chance Coincidence}

To assess which galaxy is the most probable host of GRB 111020A, we adopt the methodology of Bloom et al. (2002) and Berger (2010) to calculate the probability of chance coincidence $P(<\delta R)$ at a given angular separation $\delta R$. We determine the expected number density of galaxies brighter than a measured apparent magnitude, $m$, using the results of deep optical galaxy surveys (Hogg et al. 1997; Beckwith et al. 2006):

$$
\sigma(\leqslant m)=\frac{1}{0.33 \times \ln (10)} \times 10^{0.33(m-24)-2.44} \operatorname{arcsec}^{-2} .
$$

Then the probability of chance coincidence is given by (Bloom et al. 2002)

$$
P(<\delta R)=1-e^{-\pi(\delta R)^{2} \sigma(\leqslant m)} .
$$

We calculate $P(<\delta R)$ for each of the three host galaxy candidates (Figure 3 ) and find that G3 is the most probable host of GRB 111020 A with $P(<\delta R)=0.01$, while for $\mathrm{G} 1$ and G2 the values are $P(<\delta R)=0.10$ and 0.12 , respectively. 


\subsection{X-Ray Light-curve Fitting and a Jet Break}

The temporal behavior of the X-ray afterglow flux is characterized by a steady power-law decline until $\delta t \approx 2$ days, when there is a significant steepening in the light curve (Figure 1). A single power-law model with a decline rate determined by the $\mathrm{X}$-ray data at early times ( $t \lesssim 2$ days) provides a poor fit to the late-time data (dotted line in Figure 1); in particular, it overestimates the Chandra detection and upper limit. To quantitatively assess the shape of the X-ray light curve, we therefore invoke a broken power-law model, given by

$$
F_{X}=F_{X, 0}\left[\left(\frac{t}{t_{j}}\right)^{\alpha_{X, 1} s}+\left(\frac{t}{t_{j}}\right)^{\alpha_{X, 2} s}\right]^{1 / s},
$$

where $F_{X, 0}=2^{1 / s} F_{X}\left(t=t_{j}\right), \alpha_{X, 1}$ and $\alpha_{X, 2}$ are the powerlaw indices pre- and post-break, respectively, $t_{j}$ is the break time in seconds, and $s$ is a dimensionless smoothness parameter that characterizes the sharpness of the break. We perform a three-parameter $\chi^{2}$-grid search over $F_{X, 0}, \alpha_{X, 1}$ and $t_{j}$. If we use a relatively sharp break (e.g., $s \approx-10$ ), the Chandra $3 \sigma$ upper limit constrains $\alpha_{X, 2} \lesssim-1.7$. If we allow for a smoother break (e.g., $s \approx-1$ ), $\alpha_{X, 1}$ remains unchanged but the break occurs at later times $\left(t_{j} \approx 4\right.$ days $)$ and $\alpha_{X, 2}$ is required to have a steeper value of $\lesssim-2.2$ to accommodate the Chandra upper limit. This scenario generally provides a poorer fit to the last Chandra and Swift/XRT points. We therefore adopt the sharp-break scenario. Fixing $s=-10$ and $\alpha_{X, 2}=-2.1$, we find a best-fit broken power-law model characterized by $F_{X}\left(t_{j}\right)=(1.36 \pm 0.45) \times 10^{-13} \mathrm{erg} \mathrm{cm}^{-2} \mathrm{~s}^{-1}$, $\alpha_{X, 1}=-0.78 \pm 0.05$, and $t_{j}=2.0 \pm 0.5$ days $\left(1 \sigma, \chi_{v}^{2}=1.1\right.$ with 15 dof, Figure 1). This best-fit model is shown in Figure 1. The best-fit parameters are independent of our choice of $\alpha_{X, 2}$ between -1.7 and -3 . We also note the presence of a slight flux enhancement relative to the power-law decay at $\delta t \approx 3 \times 10^{4} \mathrm{~s}$ ( 0.35 days). If we remove these points from our fits, the resulting best-fit parameters remain unaffected.

The required change in the temporal index is $\Delta \alpha_{12} \gtrsim 0.9$. There are several possibilities that can explain breaks in GRB afterglow light curves. The first scenario is the transition of the cooling frequency across the band, but this only predicts $\Delta \alpha=0.25$ (Sari et al. 1998). An alternative possibility is the cessation of energy injection, from either refreshed shocks or a long-lasting central engine (e.g., Rees \& Meszaros 1998; Sari \& Mészáros 2000; Zhang \& Mészáros 2002), which has been used to explain the termination of a shallow decay or plateau phase in the X-ray and optical light curves of several long GRBs. However, these cases all exhibit earlier temporal breaks at $\sim 10^{3}-10^{4}$ s with $\Delta \alpha_{12} \sim 0.7\left(\alpha_{X, 1} \approx-0.5, \alpha_{X, 2} \approx-1.2\right.$; Nousek et al. 2006; Zhang et al. 2006; Liang et al. 2007). Attributing the break in GRB 111020A to the cessation of central engine activity would require sustained energy injection from the start of XRT monitoring to the break time, $\sim 100 \mathrm{~s}$ to 2 days, whereas the timescales of energy injection for long GRBs are $\lesssim$ few hours (Nousek et al. 2006; Zhang et al. 2006; Liang et al. 2007; Racusin et al. 2009). Single episodes of energy injection have also been observed in two short GRBs: 051221A and 050724A (Berger et al. 2005; Soderberg et al. 2006; Burrows et al. 2006; Grupe et al. 2006)). The light curve of GRB 051221A, which exhibits a power-law decay with index $\alpha_{X, 1}=-1.1$, a plateau, and a return to the same power law $\left(\Delta \alpha_{12}=0\right)$, is interpreted as a single period of energy injection (Soderberg et al. 2006; Burrows et al. 2006). A superimposed flare on the light curve of GRB 050724A with a single underlying decay index of $\alpha_{X, 1}=-0.98$ is also possibly related to late-time reactivation of the central engine (Berger et al. 2005; Grupe et al. 2006; Figure 1). Neither of these light curves resembles the behavior of GRB 111020A, where the change in slope is substantially greater.

Yet another possibility to explain the break is a sharp change in the external density. However, models for density jumps in a uniform medium (Nakar \& Granot 2007) predict that the density would need to decrease by greater than a factor of $\sim 10^{3}$ to account for the observed $\Delta \alpha_{12}>0.9$ steepening. More realistic density contrasts of $\sim 10$ predict $\Delta \alpha_{\max } \approx 0.4$ in optical and X-ray afterglow light curves (Nakar \& Granot 2007).

Finally, we consider that the observed steepening is a jet break, when the edge of a relativistically beamed outflow becomes visible to the observer and the jet spreads laterally (Sari et al. 1999; Rhoads 1999). This model is often adopted to explain $\Delta \alpha_{12} \sim 1$ in the light curves of long GRBs (e.g., Frail et al. 2001; Bloom et al. 2003; Racusin et al. 2009) and has been observed in one other short burst, GRB 051221A $\left(\Delta \alpha_{12} \sim 0.9\right.$, Figure 1; Soderberg et al. 2006; Burrows et al. 2006). Given the similarity in $\Delta \alpha_{12}$ and the timescales of jet breaks in both short and long GRBs, we conclude that the observed steepening in the light curve of GRB 111020A is best explained by a jet break at $t_{j}=2.0 \pm 0.5$ days.

\subsection{Afterglow Properties}

We utilize our radio, optical, and X-ray observations to constrain the explosion properties and circumburst environment of GRB 111020A. In particular, we adopt the standard synchrotron model for GRB afterglows (Sari et al. 1998; Granot \& Sari 2002), which provides a mapping from observable properties to the isotropic-equivalent kinetic energy $\left(E_{\mathrm{K} \text {,iso }}\right)$, circumburst density $\left(n_{0}\right)$, and the fractions of post-shock energy in radiating electrons $\left(\epsilon_{e}\right)$ and magnetic fields $\left(\epsilon_{B}\right)$. We use data at the time of the radio and first optical observations $(\delta t=17.7 \mathrm{hr})$, as well as the decay indices from the full X-ray light curve.

First, we constrain the electron power-law index $p$, using a combination of temporal and spectral information. From the $\mathrm{X}$-ray light curve, we measure $\alpha_{X, 2} \lesssim-1.7$ (Section 3.2). For $p=-\alpha_{X, 2}$, appropriate for a spreading jet (Sari et al. 1999), we can then constrain $p \gtrsim 1.7$. To further constrain $p$ and investigate the location of the cooling frequency, $v_{c}$, we compare the values $\alpha_{X, 1}=-0.78 \pm 0.05$ and $\beta_{X}=-1.04 \pm$ $0.16\left(\beta_{X}=1-\Gamma ; 1 \sigma\right)$ to the closure relations for a relativistic blast wave in a constant-density interstellar-mediumlike medium for $p>2$, a typical environment expected for a short GRB from a non-massive star progenitor (Sari et al. 1999; Granot \& Sari 2002). If $v_{c}>v_{X}$, then the independently derived values for $p$ from the temporal and spectral indices are inconsistent: $p=2.0 \pm 0.07$ from $\alpha_{X, 1}$, and $p=3.1 \pm 0.32$ from $\beta_{X}$ (errors are $1 \sigma$ ).

However, if $v_{c}<v_{X}$, we obtain $p=1.7 \pm 0.07$ from $\alpha_{X, 1}$ (Granot \& Sari 2002), which is consistent with the $p$ value inferred from $\alpha_{X, 2}$ but yields a divergent total integrated energy in electrons unless a break at high energies in the distribution is invoked. Although a flat electron distribution $(p<2)$ is possible and not uncommon (e.g., Dai \& Cheng 2001; Panaitescu \& Kumar 2001; Racusin et al. 2009), the standard relations for $1<p<2$ yield $p=0.84 \pm 0.25$ from $\alpha_{X, 1}$. This solution is not self-consistent and would also require an unusually sharp break of $\Delta p \gtrsim 1.2$ in the electron distribution. Continuing with the assumptions that $v_{c}<v_{X}$ and $p>2$, we obtain $p=2.1 \pm 0.32$ from $\beta_{X}$, which is marginally consistent with 

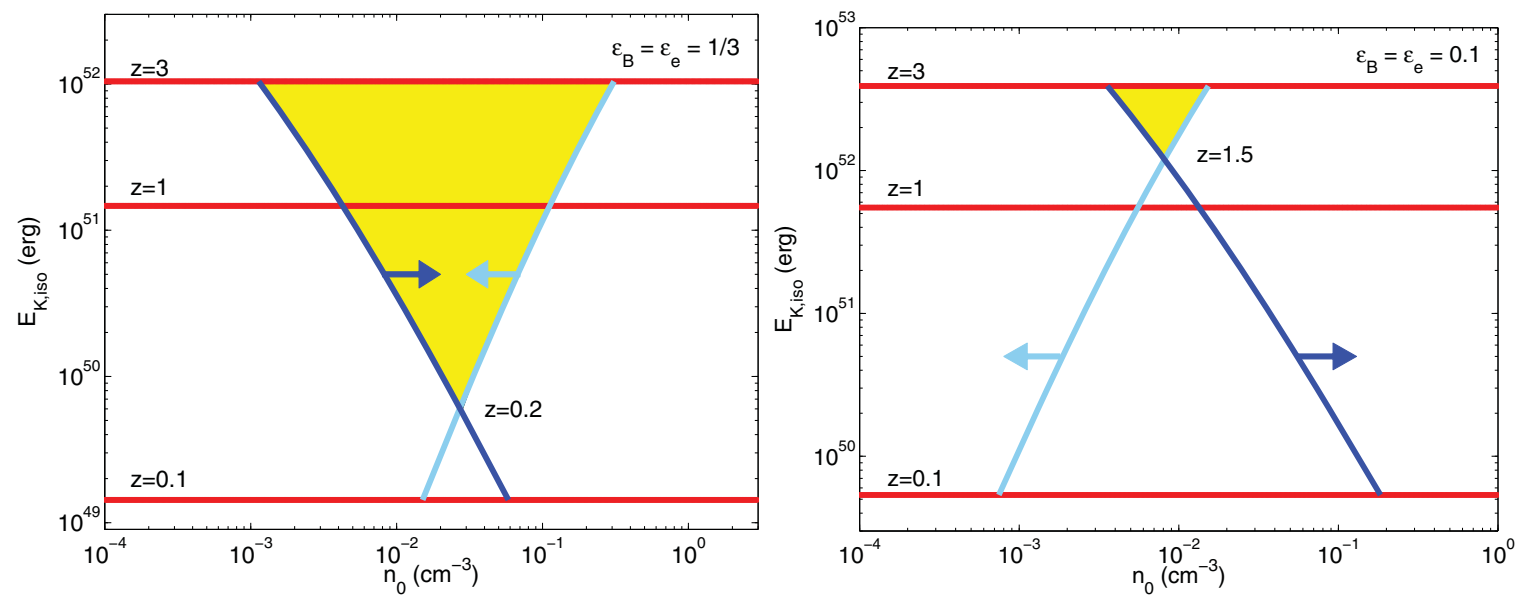

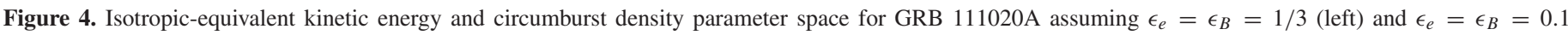

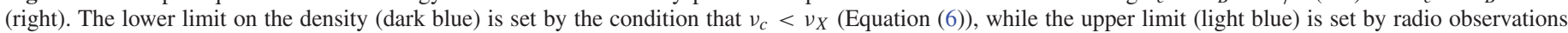
(Equation (5)). Also plotted are the values for $E_{\mathrm{K} \text {,iso }}$ at $z=0.1,1$, and 3 (red). The allowable parameter space set by these constraints is filled in yellow.

(A color version of this figure is available in the online journal.)

the value inferred from the temporal index. Put another way, $\alpha-3 \beta / 2=0.77 \pm 0.30$, which satisfies the closure relation for $v_{c}<v_{X}$ (Sari et al. 1998). We therefore conclude that $v_{c}<v_{X}$. We note that the spectral index is generally more reliable in the determination of $p$ because it remains constant over time and is not subject to alternative processes such as energy injection or flaring. In this case, the same $\beta_{X}$ was also independently determined from both the XMM and XRT data sets (Table 1). Therefore, for the rest of our calculations, we take a reasonable value of $p=2.1$ as determined from $\beta_{X}$.

We next determine a set of constraints on $n_{0}$ and $E_{\mathrm{K} \text {,iso }}$ based on the X-ray flux density, radio limit, and the condition that $v_{c}<v_{X}$. First, we use the X-ray afterglow emission as a proxy for $E_{\mathrm{K} \text {,iso }}$ assuming that the X-ray emission is from the forward shock. For $v_{c}<v_{X}$ at the time of our broadband observations $(\delta t=17.7 \mathrm{hr})$, we use $F_{X}=0.032 \mu \mathrm{Jy}$ at $\nu_{X}=2.4 \times 10^{17} \mathrm{~Hz}$ $(1 \mathrm{keV}$ ) and $p=2.1$ to obtain (Granot \& Sari 2002)

$$
E_{\mathrm{K}, \text { iso }} \approx 2.2 \times 10^{50}(1+z)^{-1} \epsilon_{e}^{-1.07} \epsilon_{B}^{-0.024} d_{\mathrm{L}, 28}^{1.95} \mathrm{erg},
$$

where $d_{\mathrm{L}, 28}$ is the luminosity distance in units of $10^{28} \mathrm{~cm}$. Next, we use $E_{\mathrm{K} \text {,iso }}$ to constrain $n_{0}$. Using our $3 \sigma$ EVLA limit of $F_{v \text {,rad }} \lesssim 39 \mu \mathrm{Jy}$, we can determine an upper limit on $n_{0}$ under the reasonable assumption that our observed radio band $(v=5.8 \mathrm{GHz})$ is above the self-absorption frequency $\left(v_{a}<v_{\text {rad }}<v_{m}, F_{v, \text { rad }} \propto n_{0}^{1 / 2}\right)$ at the time of observations. For this scenario (Granot \& Sari 2002),

$$
n_{0} \lesssim 1.7 \times 10^{-3} E_{\mathrm{K}, \text { iso }, 52}^{-5 / 3}(1+z)^{-5 / 3} d_{L, 28}^{4} \epsilon_{e}^{4 / 3} \epsilon_{B}^{-2 / 3} \mathrm{~cm}^{-3},
$$

where $E_{\mathrm{K}, \text { iso, } 52}$ is in units of $10^{52} \mathrm{erg}$ and $n_{0}$ is in $\mathrm{cm}^{-3}$. As noted in Section 2.4, if the marginal radio detection is indeed real, then this upper bound can be replaced with an equality. Finally, we can place a lower limit on the density using the condition that $v_{c}<v_{X}$ (i.e., $\left.v_{c} \lesssim 2.4 \times 10^{16} \mathrm{~Hz} ; 0.1 \mathrm{keV}\right)$ :

$$
n_{0} \gtrsim 4.5 \times 10^{-4}(1+z)^{-1 / 2} \epsilon_{B}^{-3 / 2} E_{\mathrm{K}, \text { iso }, 52}^{-1 / 2} \mathrm{~cm}^{-3} .
$$

It is clear that $E_{\mathrm{K} \text {,iso }}$ and $n_{0}$ depend sensitively on our choice of $z, \epsilon_{e}$, and $\epsilon_{B}$. The fractions $\epsilon_{e}, \epsilon_{B}$ are not expected to exceed

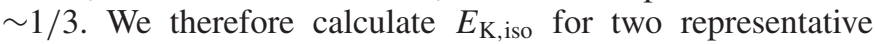
cases: $\epsilon_{e}=\epsilon_{B}=1 / 3$ (Case I), and more typical values of
$\epsilon_{e}=\epsilon_{B}=0.1$ (Case II). We then calculate the range of allowed $n_{0}$ set by Equations (5) and (6), which becomes wider as the redshift increases. ${ }^{10}$ For Case $\mathrm{I}$, this requires that $z \gtrsim 0.2$, below which the constraints on $n_{0}$ conflict (Figure 4). At the median observed redshift of the short GRB population, $z \sim 0.5$, we obtain $E_{\mathrm{K} \text {,iso }} \approx 3.7 \times 10^{50} \mathrm{erg}$ and $n_{0}=0.01-0.06 \mathrm{~cm}^{-3}$. For Case II, the constraints on $n_{0}$ require a higher redshift of $z \gtrsim 1.5$ (Figure 4). For a fiducial redshift of $z=1.5$, we obtain $E_{\mathrm{K} \text {,iso }} \approx 1.2 \times 10^{52} \mathrm{erg}$ and $n_{0}=0.008 \mathrm{~cm}^{-3}$. The parameters for the two cases are listed in Table 4. Although we cannot distinguish between these two scenarios, both cases require low circumburst densities of $n \sim 0.01-0.1 \mathrm{~cm}^{-3}$.

\subsection{Jet Opening Angle}

In the context of a jet break, we use the time of the break from the X-ray light curve ( $2.0 \pm 0.5$ days $)$ and the circumburst density and energy estimates from the previous section to constrain $\theta_{j}$. The time of the break is a direct reflection of the jet opening angle (Sari et al. 1999; Frail et al. 2001),

$$
\theta_{j}=0.1 t_{j, \mathrm{~d}}^{3 / 8}(1+z)^{-3 / 8} E_{\mathrm{K}, \text { iso }, 52}^{-1 / 8} n_{0}^{1 / 8},
$$

where $t_{j, \mathrm{~d}}$ is expressed in days. For our fiducial Case I $(z=$ $\left.0.5, \epsilon_{e}=\epsilon_{B}=1 / 3\right), E_{\mathrm{K}, \text { iso }} \approx 3.7 \times 10^{50} \mathrm{erg}$ and $n \approx$ $0.01-0.06 \mathrm{~cm}^{-3}$ give $\theta_{j}=7^{\circ}-8^{\circ}$. This leads to a beaming correction on the energy of $f_{b} \equiv\left[1-\cos \left(\theta_{j}\right)\right]=0.007-0.01$ and therefore a true kinetic energy $E_{K}=f_{b} E_{\mathrm{K} \text {,iso }} \approx(3-4) \times$ $10^{48}$ erg (Table 4). To estimate the beaming-corrected $\gamma$-ray energy, we infer $E_{\gamma, \text { iso }}$ from the Swift/BAT fluence and apply a bolometric correction factor of five to roughly convert to a representative observed $\gamma$-ray energy range of $\sim 10-1000 \mathrm{keV}$. This factor is derived from short GRBs observed by satellites with wider energy coverage (Berger 2010; Margutti et al. 2012). We obtain $E_{\gamma, \text { iso }}=2.1 \times 10^{50}$ erg and therefore a true $\gamma$-ray energy of $E_{\gamma} \approx 2 \times 10^{48} \mathrm{erg}$.

For Case II $\left(z=1.5, \epsilon_{e}=\epsilon_{B}=0.1\right)$, where $n_{0} \approx$ $0.008 \mathrm{~cm}^{-3}$ and $E_{\mathrm{K}, \text { iso }} \approx 1.2 \times 10^{52} \mathrm{erg}$, we obtain a smaller

\footnotetext{
10 Assuming non-equipartition in two alternative cases, $\epsilon_{B}=0.01, \epsilon_{e}=0.1$ and $\epsilon_{B}=0.1, \epsilon_{e}=0.01$, our constraints on $n_{0}$ conflict unless $z \gtrsim 3$ and result in $n_{0} \sim 0.01-1 \mathrm{~cm}^{-3}$. Based on our observations of the putative host galaxies and the typical luminosity of short GRB hosts, we find a high- $z$ origin unlikely (see Section 4.2).
} 
Table 4

Physical Parameters of GRB 111020A

\begin{tabular}{lcc}
\hline \hline Parameter & Case I $\left[z=0.5, \epsilon_{e}=\epsilon_{B}=1 / 3\right]$ & Case II $\left[z=1.5, \epsilon_{e}=\epsilon_{B}=0.1\right]$ \\
\hline$t_{j}$ & $2.0 \pm 0.5 \mathrm{days}^{\mathrm{a}}$ & $2.0 \pm 0.5 \mathrm{days}^{\mathrm{a}}$ \\
$E_{\gamma, \text { iso }}$ & $2.1 \times 10^{50} \mathrm{erg}$ & $1.9 \times 10^{51} \mathrm{erg}$ \\
$E_{\mathrm{K}, \text { iso }}$ & $3.7 \times 10^{50} \mathrm{erg}^{-3}$ & $1.2 \times 10^{52} \mathrm{erg}^{-3}$ \\
$n_{0}$ & $0.01-0.06 \mathrm{~cm}^{-3}$ & $0.008 \mathrm{~cm}^{-3}$ \\
$\theta_{j}$ & $7^{\circ}-8^{\circ}$ & $3^{\circ}$ \\
$f_{b}$ & $0.007-0.01$ & 0.001 \\
$E_{\gamma}$ & $2 \times 10^{48} \mathrm{erg}$ & $3 \times 10^{48} \mathrm{erg}$ \\
$E_{K}$ & $(3-4) \times 10^{48} \mathrm{erg}$ & $2 \times 10^{49} \mathrm{erg}$ \\
$E_{\mathrm{tot}}$ & $(5-6) \times 10^{48} \mathrm{erg}$ & $2 \times 10^{49} \mathrm{erg}$ \\
$\eta_{\gamma}$ & $0.3-0.4$ & 0.15
\end{tabular}

Note. ${ }^{\text {a }}$ Uncertainties correspond to a $1 \sigma$ confidence level.

opening angle of $\theta_{j} \approx 3^{\circ}$. This leads to $f_{b} \approx 1.4 \times 10^{-3}$ and hence $E_{\gamma} \approx 3 \times 10^{48} \mathrm{erg}$ and $E_{K} \approx 2 \times 10^{49} \mathrm{erg}$.

In both cases, the true $\gamma$-ray energy is a few $\times 10^{48} \mathrm{erg}$, while the kinetic energy is an order of magnitude higher at $z=1.5$ than at $z=0.5$. This results in a lower $\gamma$-ray conversion efficiency $\left(\eta_{\gamma} \equiv E_{\gamma} / E_{\text {tot }}\right)$ for Case II of 0.15 compared to 0.3-0.4 for Case I (Table 4). The total energy even for Case II is $\sim 10-100$ times lower that for long GRBs.

\subsection{Extinction}

We investigate the presence of extinction by comparing the unabsorbed X-ray flux and the optical non-detection at $\delta t=17.7 \mathrm{hr}$. Since we do not know the exact location of the cooling frequency, we assume a maximum value $v_{c, \max }$ of $2.4 \times 10^{17} \mathrm{~Hz}(1 \mathrm{keV})$ and extrapolate the X-ray flux to the optical band using the shallowest possible slope of $\beta=-(p-1) / 2=-0.55$ to obtain the lowest bound on the expected optical afterglow flux in the absence of extinction; any other assumption for the location of $v_{c}<v_{X}$ would result in a higher predicted optical flux density. For $p=2.1$ we estimate $F_{v, \text { opt }} \approx 1.1 \mu \mathrm{Jy}(i=23.8 \mathrm{mag})$. Given that our observed $3 \sigma$ upper limit is $i \gtrsim 24.4 \mathrm{mag}$, this implies a lower limit on the optical extinction in excess of the Galactic value of $A_{i} \gtrsim 0.6$ mag. ${ }^{11}$ In the rest frame of the burst for a Milky Way extinction curve, this translates to $A_{V}^{\text {host }} \gtrsim 0.6$ mag for $z=0.5$ and $A_{V}^{\text {host }} \gtrsim 0.2$ at $z=1.5$ (Cardelli et al. 1989). Using Galactic relations between $N_{H}$ and $A_{V}, N_{\mathrm{H}, \text { int }} / A_{V} \approx(1.7-2.2) \times 10^{21}$ (Predehl \& Schmitt 1995; Watson 2011), we infer lower limits of $N_{\mathrm{H}, \text { int }} \gtrsim 10^{21} \mathrm{~cm}^{-2}$ at $z=0.5$ and $N_{\mathrm{H} \text {,int }} \gtrsim 4.4 \times 10^{20} \mathrm{~cm}^{-2}$ at $z=1.5$, consistent with our value of $7.5 \times 10^{21} \mathrm{~cm}^{-2}$ $(z=0)$ derived from the X-ray spectrum (Table 1$)$. However, an appreciable extinction is unexpected given the burst's location on the outskirts of its potential host galaxy. We note that the burst is located at Galactic coordinates $(l, b)=\left(359^{\circ} .3,-19.4\right)$, which is toward the Galactic bulge on a steep gradient in the dust map (Schlegel et al. 1998) and thus may be subject to substantial $(\sim 30 \%)$ uncertainties in the Galactic extinction. ${ }^{12}$ Taking this uncertainty into account reduces the required $A_{V}^{\text {host }}$ to $\gtrsim 0.2-0.3$ mag depending on the redshift of the burst.

\footnotetext{
11 We note that for $p \lesssim 1.9$, no host galaxy extinction is required.

12 Using a high-resolution $\left(\theta_{\text {FWHM }}=15^{\prime \prime}\right)$ WISE $12 \mu \mathrm{m}$ map, we do not see strong evidence for any thin-dust filaments at the location of the burst that would result in $>30 \%$ uncertainties in the Galactic $A_{V}$ (D. Finkbeiner 2012, private communication).
}

\section{DISCUSSION}

\subsection{Environment}

From our broadband observations, we constrain the circumburst density of GRB $111020 \mathrm{~A}$ to $n_{0} \sim 0.01-0.1 \mathrm{~cm}^{-3}$, which is consistent with the low values inferred for a few previous short GRBs (Soderberg et al. 2006; Panaitescu 2006; Stratta et al. 2007; Perley et al. 2009b; Berger 2010; Fong et al. 2011). The inferred density fits well with the framework of NS-NS/NS-BH binary progenitor systems, which may be subject to substantial kicks from their host galaxies and are predicted to typically occur at densities of $\sim 10^{-6}$ to $1 \mathrm{~cm}^{-3}$ (Perna \& Belczynski 2002; Belczynski et al. 2006).

GRB 111020 A has an offset of $\approx 0$ '.80 from its most probable host galaxy (G3, Figure 2). For redshifts between $z=$ 0.5 and 1.5, this translates to a projected physical offset of $\approx 5-7 \mathrm{kpc}$, which is comparable to the median value of $\sim 5 \mathrm{kpc}$ for well-localized short GRBs with host associations (Fong et al. 2010; Church et al. 2011). Although G3 has the lowest probability of chance coincidence by an order of magnitude (Figure 3), we cannot rule out the possibility that G3 is a faint star. The next most probable galaxies, G1 and G2, are situated 2".8 (17-24 kpc) and 6".5 (40-56 kpc), respectively, from GRB 111020A (Figure 2). If the burst originated from one of these galaxies, this would put GRB 111020A at the high end of the observed offset distribution, similar to the growing subclass of apparently "hostless" short GRBs, which likely occur $\gtrsim 30 \mathrm{kpc}$ from their host galaxies (Berger 2010). All of these inferred offsets are consistent with predicted offset distributions of NS-NS/NS-BH binaries originating in Milky-Way-type galaxies (Bloom et al. 1999; Fryer et al. 1999; Belczynski et al. 2006; Salvaterra et al. 2010).

Most short GRB host galaxies with confirmed spectroscopic redshifts have measured luminosities of $L_{B} \approx 0.1-1 L_{*}$ (Berger et al. 2007). The apparent magnitude of $\mathrm{G} 3$ is $i \approx 24.3$, which corresponds to $L_{B} \approx 0.1-1 L_{*}$ over $z \approx 0.5-2.3$ when compared to the luminosity function of galaxies at corresponding redshifts in the DEEP2 and LBG surveys (Willmer et al. 2006; Reddy \& Steidel 2009). This is consistent with the redshift range inferred from the afterglow.

We next investigate the nature of the dust and gas in the environment of GRB 111020A through an analysis of $A_{V}^{\text {host }}$ and $N_{\mathrm{H} \text {,int }}$. We have shown that the burst requires dust extinction of $A_{V}^{\text {host }} \gtrsim 0.2-0.6 \mathrm{mag}$, depending on the redshift of the burst and the uncertainty in Galactic extinction. We have also measured a neutral hydrogen column density intrinsic to the burst environment of $N_{\mathrm{H}, \text { int }}=(7.5 \pm 2.0) \times 10^{21} \mathrm{~cm}^{-2}$ at $z=0$, 


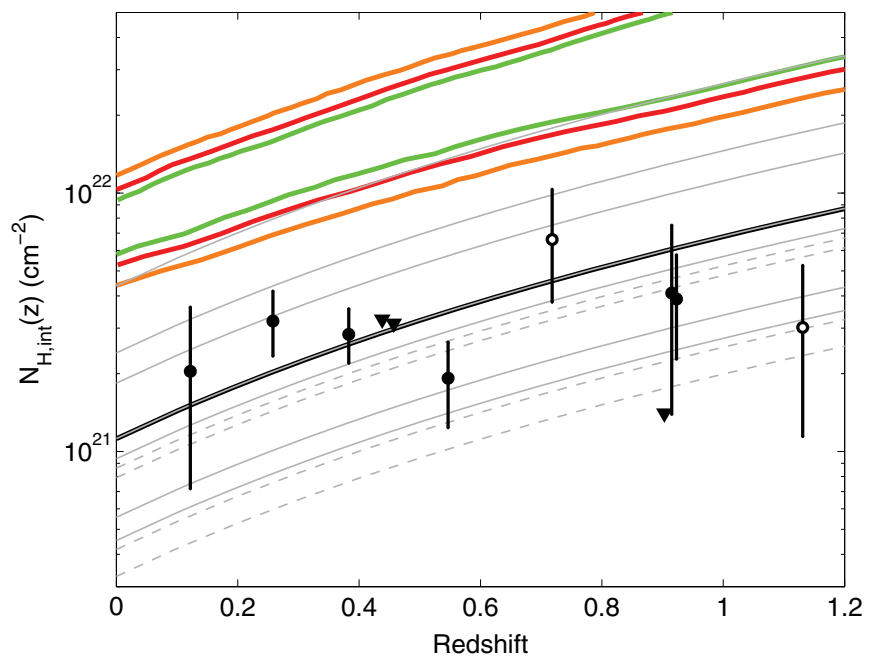

Figure 5. Excess neutral hydrogen column density, $N_{\mathrm{H}, \text { int }}$, vs. redshift for GRB 111020A $(1 \sigma, 2 \sigma$, and $3 \sigma$ intervals denoted by green, red, and orange lines) along with six Swift short GRBs with measured redshifts and optical afterglows (black filled circles) and two (GRBs 060801 and 101219A) with only X-ray afterglows (open circles). Also plotted are 11 short GRBs without secure redshifts (gray lines), 4 of which have only upper limits on $N_{\mathrm{H} \text {,int }}$ (gray

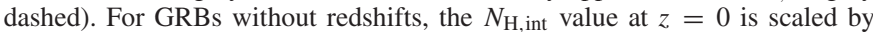
$(1+z)^{2.6}$ (Galama \& Wijers 2001). Errors and upper limits are at the $90 \%$ confidence level. The weighted mean for all short GRBs (black line) over the redshift interval $z=0-1.2$ is also shown. GRB $111020 \mathrm{~A}$ has the highest $N_{\mathrm{H} \text {,int }}$ of a short GRB to date and is well above the mean for short GRBs.

(A color version of this figure is available in the online journal.)

which becomes higher for any other choice of $z$. High values of both dust extinction and X-ray absorption have been linked to “dark" GRBs (e.g., Perley et al. 2009a; Campana et al. 2012), which have optically sub-luminous afterglows compared to their $\mathrm{X}$-ray or near-IR (NIR) counterparts and can quantitatively be classified by $\left|\beta_{O X}\right| \lesssim\left|\beta_{X}\right|-0.5$ (van der Horst et al. 2009; see also Jakobsson et al. 2004). With $\left|\beta_{X}\right|=1.0$ and $\left|\beta_{O X}\right| \lesssim 0.46$, GRB 111020A is consistent with this definition of dark GRBs. While optical extinction intrinsic to long GRB environments is not uncommon and commensurate with their origin in dusty, star-forming regions, evidence for substantial extinction has been reported for only one other short burst, GRB 070724A, which required $A_{V}^{\text {host }} \gtrsim 2$ mag to explain the suppression of optical emission relative to the NIR (Berger et al. 2009; Kocevski et al. 2010). The location of GRB 070724A on the outskirts of its host galaxy, $\sim 5 \mathrm{kpc}$ from the center, suggested either an origin in a star-forming region or a progenitor system that produced the dust itself (Berger et al. 2009). The potentially appreciable extinction and the location with respect to its putative host suggest that the same conclusions may be drawn for GRB 111020A.

On the other hand, the relation between $N_{\mathrm{H} \text {,int }}$ and the darkness of a burst is less clear. A recent study of long, dark GRBs shows them to have higher intrinsic column densities than non-dark GRBs, which suggests that the darkness of a burst is largely due to absorption by circumburst material (Campana et al. 2012). To investigate this relationship for GRB 111020A, we extract spectra and best-fitting $N_{\mathrm{H} \text {,int }}$ for all short GRBs with XRT-detected afterglows in the same manner as GRB 111020A (see Section 2.2.1), over time ranges with no evidence for spectral evolution. There are 22 short bursts with sufficient X-ray counts to perform spectral analysis, 11 of which have known redshifts (Table 5). We find a short GRB weighted average of $N_{\mathrm{H} \text {,int }}(z=0)=(1.1 \pm 0.14) \times 10^{21} \mathrm{~cm}^{-2}(90 \%$
Table 5

Intrinsic X-Ray Column Density of Hydrogen, $N_{\mathrm{H} \text {,int }}$, for Swift Short GRBs

\begin{tabular}{|c|c|c|c|}
\hline GRB & $z$ & $\begin{array}{c}N_{\mathrm{H}, \text { int }} \\
\left(10^{21} \mathrm{~cm}^{-2}\right)\end{array}$ & $\sigma$ above Zero \\
\hline 050724 & 0.258 & $3.20_{-0.86}^{+0.97}$ & 5.7 \\
\hline 051210 & $\cdots$ & $<0.54$ & \\
\hline 051221A & 0.547 & $1.92_{-0.68}^{+0.73}$ & 4.5 \\
\hline 060313 & $\cdots$ & $0.45_{-0.33}^{+0.36}$ & 2.1 \\
\hline 060801 & 1.131 & $3.02_{-1.88}^{+2.22}$ & 2.4 \\
\hline 061006 & 0.438 & $<2.04$ & \\
\hline 061201 & $\cdots$ & $0.94_{-0.53}^{+0.60}$ & 2.7 \\
\hline 070714B & 0.923 & $3.89_{-1.61}^{+1.87}$ & 4.2 \\
\hline 070724A & 0.457 & $<1.89$ & \\
\hline 071227 & 0.383 & $2.84_{-0.65}^{+0.72}$ & 6.8 \\
\hline 080123 & $\cdots$ & $1.12_{-0.26}^{+0.28}$ & 6.8 \\
\hline 080905A & 0.122 & $2.04_{-1.33}^{+1.58}$ & 2.3 \\
\hline 090510 & 0.903 & $<0.80$ & \\
\hline 090515 & $\cdots$ & $0.56_{-0.27}^{+0.30}$ & 3.2 \\
\hline 090607 & $\cdots$ & $<0.79$ & \\
\hline 091109B & $\ldots$ & $<1.58$ & \\
\hline 100117A & 0.915 & $4.10_{-2.71}^{+3.41}$ & 2.2 \\
\hline $100702 \mathrm{~A}$ & $\cdots$ & $4.37_{-3.05}^{+3.67}$ & 2.1 \\
\hline 101219A & 0.718 & $6.61_{-2.82}^{+3.73}$ & 3.3 \\
\hline $110112 \mathrm{~A}$ & $\ldots$ & $<0.92$ & \\
\hline $111020 \mathrm{~A}$ & $\cdots$ & $7.50_{-1.8}^{+2.0}$ & 6.5 \\
\hline 111117A & $\cdots$ & $1.84_{-1.05}^{+1.28}$ & 2.6 \\
\hline $111121 \mathrm{~A}$ & $\cdots$ & $2.41_{-0.74}^{+0.82}$ & 5.1 \\
\hline
\end{tabular}

Note. Errors and upper limits quoted correspond to a $90 \%$ confidence level; $z=0$ is assumed when the redshift is not known.

CL, Figure 5). In comparison, GRB $111020 \mathrm{~A}$ has a high value of $N_{\mathrm{H} \text {,int }}=(7.5 \pm 2.0) \times 10^{21} \mathrm{~cm}^{-2}$ at $z=0$ (Figure 5). Taken at face value, it is surprising to find a large $N_{\mathrm{H} \text {,int }}$ for a substantial offset and may suggest that the burst occurred in a relatively metal-rich environment.

\subsection{Beaming, Energetics, and Rates}

We uncover a break in the X-ray light curve of GRB $111020 \mathrm{~A}$ at $\approx 2$ days, which we interpret as a jet break (Section 3.2). Depending on our values for $z, \epsilon_{e}$, and $\epsilon_{B}$, we infer an opening angle of $\approx 3^{\circ}-8^{\circ}$. This is reminiscent of the first jet break discovery in GRB 051221A, with $\theta_{j} \approx 7^{\circ}$ (Soderberg et al. 2006; Burrows et al. 2006), and suggests that at least a fraction of these events are highly collimated. In addition, temporal breaks at $t_{j} \lesssim$ few hours in GRBs 061201 (Stratta et al. 2007) and 090510 (De Pasquale et al. 2010; Nicuesa Guelbenzu et al. 2012), if interpreted as jet breaks, lead to $\theta_{j} \approx 1^{\circ}$ (Figure 6). However, these two cases resemble early breaks in long GRBs that are generally attributed to the cessation of energy injection, and not collimation.

Although the remaining short GRB afterglow data are sparse, the lack of observed jet breaks in their X-ray light curves can be used to place lower limits on the opening angles. Indeed, Chandra observations of GRB 050724A out to 22 days indicated $\theta_{j} \gtrsim 25^{\circ}$, consistent with a spherical explosion (Grupe et al. 2006). A recent study by Coward et al. (2012) analyzed the sample of short GRB Swift/XRT light curves up to 2011 August with monitoring $\gtrsim 1$ day, which included six additional events and inferred $\theta_{j} \gtrsim 6^{\circ}-16^{\circ}$, assuming $n_{0}=1 \mathrm{~cm}^{-3}$ for all bursts. 


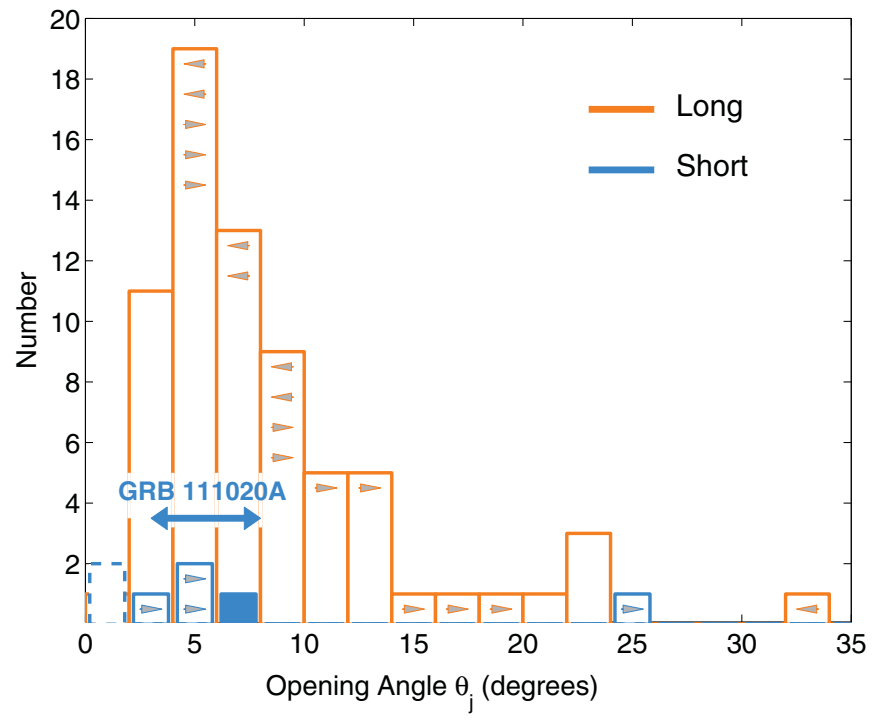

Figure 6. Distribution of opening angles for long (orange) and short (blue) GRBs. Arrows represent upper and lower limits. The long GRB population includes pre-Swift (Frail et al. 2001; Berger et al. 2003; Bloom et al. 2003; Ghirlanda et al. 2004; Friedman \& Bloom 2005), Swift (Racusin et al. 2009; Filgas et al. 2011), and Fermi (Cenko et al. 2010, 2011; Goldstein et al. 2011) bursts. The opening angle for GRB $111020 \mathrm{~A}$ ranges from $\sim 3^{\circ}$ to $8^{\circ}$ (depending on the redshift), while GRB 051221A has $\theta_{j} \approx 7^{\circ}$ (Soderberg et al. 2006; Burrows et al. 2006). Tentative jet breaks (blue dashed) for GRBs 061201 (Stratta et al. 2007) and 090510 (De Pasquale et al. 2010; Nicuesa Guelbenzu et al. 2012) are at $\sim 1^{\circ}$. Short GRB lower limits are from the non-detection of jet breaks in Swift/XRT data (this work; revised from Coward et al. 2012) and Chandra data for GRB 050724A (Berger et al. 2005; Grupe et al. 2006).

(A color version of this figure is available in the online journal.)

We revise this analysis for three of the events with robust X-ray light curves (GRBs 070714B, 070724A, 071227; data analysis prescriptions from Margutti et al. 2012) employing a more representative $n_{0} \approx 10^{-2} \mathrm{~cm}^{-3}$ (e.g., Soderberg et al. 2006 and this work). We derive $E_{\gamma \text {,iso }}$ from the reported fluences, applying a bolometric correction when necessary to represent an energy range of $\sim 10-1000 \mathrm{keV}$, and infer more realistic lower limits of $\gtrsim 2^{\circ}-6^{\circ}$ (Figure 6). These limits are indeed lower than the detected values for GRBs 051221A and 111020A and therefore do not add strong constraints on the distribution. We caution that the sample presented here represents only the $\sim 30 \%$ of the Swift short GRB population that has bright X-ray afterglows and relatively slow flux decline rates; the remaining fraction does not have detectable X-ray afterglows or fades too quickly, so constraints cannot be placed on their collimation.

There are now two short GRBs with opening angle measurements, two with measurements based on more tentative early breaks, and an additional four with lower limits (Figure 6). These early constraints create a distribution that may mimic the distribution for long GRBs, which ranges from $\sim 2^{\circ}$ to $20^{\circ}$ with a median of $7^{\circ}$ (Figure 6; Frail et al. 2001; Berger et al. 2003; Bloom et al. 2003; Ghirlanda et al. 2004; Friedman \& Bloom 2005; Racusin et al. 2009; Cenko et al. 2010; Filgas et al. 2011; Goldstein et al. 2011; Cenko et al. 2011). More events are needed to assess the real differences between the distributions. However, simulations of post-merger black hole accretion predict jets with $\theta_{j} \sim 5^{\circ}-20^{\circ}$ (Aloy et al. 2005; Rosswog 2005; Rezzolla et al. 2011) to several tens of degrees (Ruffert \& Janka 1999b; Rezzolla et al. 2011) depending on the mechanism of energy extraction and Lorentz factor, so there are expectations on theoretical grounds that the short GRB distribution is wider.
The first major ramification of collimation is the correction to the total energy release: the true energy is lower than the isotropic-equivalent value by the beaming factor, $f_{b}$. For GRB $111020 \mathrm{~A}$ with an opening angle of $\approx 3^{\circ}-8^{\circ}$, this correction factor is substantial, 0.001-0.01. Depending on the redshift, the beaming-corrected energy of GRB 111020A is $E_{\gamma} \approx(2-3) \times 10^{48}$ erg (Table 4), which is an order of magnitude lower than for GRB 051221A, with $E_{\gamma} \approx(1-2) \times 10^{49} \mathrm{erg}$ (Soderberg et al. 2006; Burrows et al. 2006), and GRB 050724A, with $E_{\gamma} \approx(0.4-4) \times 10^{50}$ erg (Grupe et al. 2006). The three remaining events with opening angle lower limits, GRBs 070714B, 070724A, and 071227, have ranges of $E_{\gamma} \approx$ $10^{48}-10^{51} \mathrm{erg}$, where the upper bound is set by the isotropicequivalent $\gamma$-ray energy in the $\approx 10-1000 \mathrm{keV}$ band. The small population of short GRBs with measured $E_{\gamma}$ therefore has a median value of $E_{\gamma} \sim 10^{49} \mathrm{erg}$, which is an order of magnitude below Swift long GRBs (Kocevski \& Butler 2008; Racusin et al. 2009) and two orders of magnitude below the pre-Swift population (Frail et al. 2001; Bloom et al. 2003). Again, this sample is incomplete because we can only measure $E_{\gamma}$ for bursts with well-constrained opening angles.

In a similar vein, we compare the beaming-corrected kinetic energy and total energy $\left(E_{K}, E_{\text {tot }}\right)$ of GRB $111020 \mathrm{~A}$ to the values for other short bursts. Because $E_{\mathrm{K} \text {,iso }}$ is more sensitive to our choices for $z, \epsilon_{e}$, and $\epsilon_{B}$, we infer different values for Cases I and II. For Case I, we infer $E_{K} \approx(3-4) \times 10^{48} \mathrm{erg}$, $E_{\mathrm{tot}}=E_{\gamma}+E_{K} \approx(5-6) \times 10^{48} \mathrm{erg}$, and $\eta_{\gamma} \approx 0.3-0.4$. For Case II, we calculate $E_{K} \approx 2 \times 10^{49} \mathrm{erg}, E_{\text {tot }} \approx 2 \times 10^{49} \mathrm{erg}$, and $\eta_{\gamma} \approx 0.15$. GRB $051221 \mathrm{~A}$ had $E_{K} \approx 8 \times 10^{48} \mathrm{erg}$ and a total energy release of $\approx 2.5 \times 10^{49} \mathrm{erg}$ (Soderberg et al. 2006; Burrows et al. 2006), while GRB 050724 had a total energy of $10^{50}-10^{51} \mathrm{erg}$. With $E_{\text {tot }} \approx(0.5-2) \times 10^{49} \mathrm{erg}$, GRB $111020 \mathrm{~A}$ may be on the low end of the total energy distribution, but more events with beaming-corrected energies are needed to better characterize the distribution for short GRBs.

The true total energy release of short GRBs has strong implications on the energy extraction mechanism. Two primary mechanisms, the thermal energy release from $v \bar{v}$ annihilation in a baryonic outflow (Jaroszynski 1993; Mochkovitch et al. 1993) and MHD processes in the black hole's accretion remnant (e.g., Blandford \& Znajek 1977; Rosswog et al. 2003), give different estimates for the expected energy release. Predictions for $\nu \bar{v}$ annihilation are largely dependent on the mass of the disk and efficiency to produce pairs. Simulations of an outflow due to $v \bar{v}$ annihilation suggest that beaming-corrected total energy releases could reach $10^{48}-10^{49}$ erg (Ruffert \& Janka 1999a, 1999b; Popham et al. 1999; Rosswog 2005; Birkl et al. 2007; Lee \& Ramirez-Ruiz 2007). Higher energy releases can be obtained from MHD processes, which can produce luminosities of $\gtrsim 10^{52} \mathrm{erg} \mathrm{s}^{-1}\left(\gtrsim 10^{50} \mathrm{erg} \mathrm{s}^{-1}\right.$ when corrected for beaming; Popham et al. 1999; Rosswog et al. 2003; Lee \& Ramirez-Ruiz 2007) depending on the nature of the magnetic field amplification. While the true energy releases of GRBs 051221A and 050724A suggest that MHD processes may be powering these events (Berger et al. 2005; Grupe et al. 2006; Soderberg et al. 2006; Burrows et al. 2006), the total energy of GRB 111020A is consistent with predictions for both scenarios.

The second major consequence of beaming is that the true event rate is higher than the observed rate by the inverse of the beaming factor (i.e., $R_{\text {true }}=f_{b}^{-1} R_{\text {obs }}$ ). Thus, beaming provides essential information for understanding the relation 
to various progenitor systems and is of particular interest since the NS-NS/NS-BH merger rates, which are a critical input for estimates of Advanced LIGO gravitational wave detections, are highly uncertain (e.g., Abadie et al. 2010; Metzger \& Berger 2012). The current estimated observed short GRB volumetric rate is $\sim 10 \mathrm{Gpc}^{-3} \mathrm{yr}^{-1}$ (Nakar et al. 2006). The estimated NS-NS merger rate is much higher: $~ 200-3000 \mathrm{Gpc}^{-3} \mathrm{yr}^{-1}$ (Kalogera et al. 2004; Nakar et al. 2006).

The discrepancy in these rates can be explained if short GRBs have typical $\theta_{j} \sim 8^{\circ}\left(f_{b}^{-1} \sim 100\right.$; see also Metzger $\&$ Berger 2012). The determination of GRB 111020A's opening angle of $3^{\circ}-8^{\circ}\left(f_{b}^{-1}=100-730\right)$, along with the small but increasing sample of opening-angle constraints for short GRBs, implies that at least a fraction of these events are significantly beamed and that the true rate of short GRBs is at least $\sim 100-1000 \mathrm{Gpc}^{-3} \mathrm{yr}^{-1}$. With a few additional openingangle measurements, this value can be significantly improved. Other proposed progenitor models, e.g., WD-WD mergers or accretion-induced collapse of a WD/NS (Qin et al. 1998; Levan et al. 2006; Metzger et al. 2008), have estimated rates of $\lesssim 1000 \mathrm{Gpc}^{-3} \mathrm{yr}^{-1}$ and $\sim 0.1-100 \mathrm{Gpc}^{-3} \mathrm{yr}^{-1}$, respectively (Lee \& Ramirez-Ruiz 2007; Darbha et al. 2010), so if a large fraction of short GRBs have opening angles of $\lesssim 25^{\circ}$, these systems may not contribute significantly to the progenitor population.

\section{CONCLUSIONS AND FUTURE WORK}

We have presented observations of GRB 111020A, utilizing extensive coverage in the X-rays with Swift/XRT, XMM, and Chandra to uncover a temporal break, most naturally explained as a jet break. Our limit on the radio afterglow from EVLA, combined with the inference that $v_{c}<v_{X}$, leads to a robust range on the circumburst density of $\sim 0.01-0.1 \mathrm{~cm}^{-3}$. The jet break time of $\approx 2$ days leads to an opening angle of $3^{\circ}-8^{\circ}$, depending on the redshift and equipartition fractions, which leads to beaming-corrected energies of $E_{\gamma} \approx(2-3) \times 10^{48} \mathrm{erg}$, $E_{K} \approx(0.3-2) \times 10^{49} \mathrm{erg}$, and $E_{\mathrm{tot}} \approx(0.5-2) \times 10^{49} \mathrm{erg}$. This result, along with the previous jet break constraints for GRBs 051221A and 050724A, suggests that there may be a spread in true energy release, $\sim 10^{48}-10^{50}$ erg for short GRBs (Berger et al. 2005; Grupe et al. 2006; Soderberg et al. 2006; Burrows et al. 2006).

Furthermore, our optical observations provide a limit on the afterglow and enabled the discovery of a putative host galaxy with $i \approx 24.3 \mathrm{mag}$. A comparison of the X-ray and optical data at $\delta t=17.7 \mathrm{hr}$ provides a lower limit on the host galaxy extinction of $A_{V}^{\text {host }} \gtrsim 0.2-0.6 \mathrm{mag}$. This is consistent with the high intrinsic column density from X-ray absorption when compared to the mean for the short GRB population.

GRB 111020A demonstrates that rapid multi-wavelength follow-up is vital to our understanding of the basic properties of short GRBs: the geometry, energetics, and circumburst densities. In particular, the search for jet breaks on timescales of $\gtrsim$ few days is imperative for placing meaningful constraints on the opening angle distribution. Ideally, the detection of breaks in both optical and X-ray data leads to an unambiguous and tight constraint on the opening angle; however, optical afterglows are only detected in $\sim 30 \%$ of Swift short GRBs, while $\mathrm{X}$-ray afterglows have been detected in $\sim 70 \%$. Furthermore, optical afterglows are intrinsically faint and subject to host galaxy contamination, making long-term monitoring highly challenging. Therefore, the jet break search is optimized in the $\mathrm{X}$-ray band, where the burst is not subject to such contamination and the afterglow brightness is virtually independent of the typically low circumburst densities. The X-rays also allow for a measurement of the kinetic energy of the outflow. Deep radio limits provide additional constraints on the circumburst density and energy. The EVLA upgrade is now enabling us to probe events with relatively low energy scales of $\sim 10^{48} \mathrm{erg}$ and densities of $\lesssim 10^{-2} \mathrm{~cm}^{-2}$.

The collimation of short GRBs will undoubtedly further our knowledge of their true energetics and rates. While the former provides information on the explosion and energy extraction mechanisms, the latter is crucial for understanding the relation to various progenitor systems (e.g., NS-NS mergers). Significant improvement on the estimated short GRB observed rate of $\sim 10 \mathrm{Gpc}^{-3} \mathrm{yr}^{-1}$ (Nakar et al. 2006) will have a critical impact on estimates for coincident short GRB-gravitational wave detections in the era of Advanced LIGO/VIRGO (Abadie et al. 2010). Furthermore, a more complete knowledge of the short GRB redshift distribution will inform our understanding of the fraction of short GRBs that may originate in globular clusters, highly relevant to gravitational wave event rate estimates (Hopman et al. 2006; Salvaterra et al. 2008; Guetta \& Stella 2009). The uncertainty in the observed short GRB rate is dominated by the uncertainty in the beaming fraction, and with only a handful of short GRB opening angles measured to date, the discovery of even a few additional jet breaks in the coming years will enable significant progress.

We thank D. Finkbeiner for helpful discussions. The Berger GRB group at Harvard is supported by the National Science Foundation under grant AST-1107973. Partial support was also provided by the National Aeronautics and Space Administration through Chandra Award Number GO1-12072X issued by the Chandra X-Ray Observatory Center, which is operated by the Smithsonian Astrophysical Observatory for and on behalf of the National Aeronautics Space Administration under contract NAS8-03060. Additional support was provided by NASA/Swift AO6 grant NNX10AI24G. Observations were obtained with the EVLA (Program 10C-145) operated by the National Radio Astronomy Observatory, a facility of the National Science Foundation operated under cooperative agreement by Associated Universities, Inc. This paper includes data gathered with the $6.5 \mathrm{~m}$ Magellan Telescopes located at Las Campanas Observatory, Chile. This work is based in part on observations obtained at the Gemini Observatory, which is operated by the Association of Universities for Research in Astronomy, Inc., under a cooperative agreement with the NSF on behalf of the Gemini partnership: the National Science Foundation (United States), the Science and Technology Facilities Council (United Kingdom), the National Research Council (Canada), CONICYT (Chile), the Australian Research Council (Australia), Ministério da Ciência, Tecnologia e Inovação (Brazil), and Ministerio de Ciencia, Tecnología e Innovación Productiva (Argentina).

\section{REFERENCES}

Abadie, J., Abbott, B. P., Abbott, R., et al. 2010, Class. Quantum Grav., 27, 173001

Alard, C. 2000, A\&AS, 144, 363

Aloy, M. A., Janka, H.-T., \& Müller, E. 2005, A\&A, 436, 273

Beckwith, S. V. W., Stiavelli, M., Koekemoer, A. M., et al. 2006, AJ, 132, 1729 Belczynski, K., Perna, R., Bulik, T., et al. 2006, ApJ, 648, 1110

Berger, E. 2007, ApJ, 670, 1254

Berger, E. 2010, ApJ, 722, 1946

Berger, E., Cenko, S. B., Fox, D. B., \& Cucchiara, A. 2009, ApJ, 704, 877

Berger, E., Fox, D. B., Price, P. A., et al. 2007, ApJ, 664, 1000 
Berger, E., Kulkarni, S. R., \& Frail, D. A. 2003, ApJ, 590, 379

Berger, E., Price, P. A., Cenko, S. B., et al. 2005, Nature, 438, 988

Birkl, R., Aloy, M. A., Janka, H.-T., \& Müller, E. 2007, A\&A, 463, 51

Blandford, R. D., \& Znajek, R. L. 1977, MNRAS, 179, 433

Bloom, J. S., Frail, D. A., \& Kulkarni, S. R. 2003, ApJ, 594, 674

Bloom, J. S., Kulkarni, S. R., \& Djorgovski, S. G. 2002, AJ, 123, 1111

Bloom, J. S., Sigurdsson, S., \& Pols, O. R. 1999, MNRAS, 305, 763

Burrows, D. N., Grupe, D., Capalbi, M., et al. 2006, ApJ, 653, 468

Campana, S., Salvaterra, R., Melandri, A., et al. 2012, MNRAS, 421, 1697

Cardelli, J. A., Clayton, G. C., \& Mathis, J. S. 1989, ApJ, 345, 245

Cenko, S. B., Frail, D. A., Harrison, F. A., et al. 2010, ApJ, 711, 641

Cenko, S. B., Frail, D. A., Harrison, F. A., et al. 2011, ApJ, 732, 29

Chandra, P., \& Frail, D. A. 2011, Bull. Astron. Soc. India, 39, 451

Church, R. P., Levan, A. J., Davies, M. B., \& Tanvir, N. 2011, MNRAS, 413 , 2004

Coward, D., Howell, E., Piran, T., et al. 2012, arXiv:1206.5058

Dai, Z. G., \& Cheng, K. S. 2001, ApJ, 558, L109

Darbha, S., Metzger, B. D., Quataert, E., et al. 2010, MNRAS, 409, 846

De Pasquale, M., Schady, P., Kuin, N. P. M., et al. 2010, ApJ, 709, L146

Eichler, D., Livio, M., Piran, T., \& Schramm, D. N. 1989, Nature, 340, 126

Evans, P. A., Beardmore, A. P., Page, K. L., et al. 2007, A\&A, 469, 379

Evans, P. A., Beardmore, A. P., Page, K. L., et al. 2009, MNRAS, 397, 1177

Filgas, R., Krühler, T., Greiner, J., et al. 2011, A\&A, 526, A113

Fong, W., Berger, E., Chornock, R., et al. 2011, ApJ, 730, 26

Fong, W., Berger, E., \& Fox, D. B. 2010, ApJ, 708, 9

Fox, D. B., Frail, D. A., Price, P. A., et al. 2005, Nature, 437, 845

Frail, D. A., Kulkarni, S. R., Sari, R., et al. 2001, ApJ, 562, L55

Friedman, A. S., \& Bloom, J. S. 2005, ApJ, 627, 1

Fryer, C. L., Woosley, S. E., \& Hartmann, D. H. 1999, ApJ, 526, 152

Galama, T. J., \& Wijers, R. A. M. J. 2001, ApJ, 549, L209

Gehrels, N., Chincarini, G., Giommi, P., et al. 2004, ApJ, 611, 1005

Ghirlanda, G., Ghisellini, G., \& Lazzati, D. 2004, ApJ, 616, 331

Goldstein, A., Preece, R. D., Briggs, M. S., et al. 2011, arXiv:1101.2458

Granot, J., \& Sari, R. 2002, ApJ, 568, 820

Greisen, E. W. 2003, in Information Handling in Astronomy-Historical Vistas, ed. A. Heck (Astrophysics and Space Science Library, Vol. 285; Dordrecht: Kluwer), 109

Grupe, D., Burrows, D. N., Patel, S. K., et al. 2006, ApJ, 653, 462

Guetta, D., \& Stella, L. 2009, A\&A, 498, 329

Hogg, D. W., Pahre, M. A., McCarthy, J. K., et al. 1997, MNRAS, 288, 404

Hopman, C., Guetta, D., Waxman, E., \& Portegies Zwart, S. 2006, ApJ, 643, L91

Jakobsson, P., Hjorth, J., Fynbo, J. P. U., et al. 2004, ApJ, 617, L21

Jaroszynski, M. 1993, Acta Astron., 43, 183

Kalberla, P. M. W., Burton, W. B., Hartmann, D., et al. 2005, A\&A, 440, 775

Kalogera, V., Kim, C., Lorimer, D. R., et al. 2004, ApJ, 601, L179

Kocevski, D., \& Butler, N. 2008, ApJ, 680, 531

Kocevski, D., Thöne, C. C., Ramirez-Ruiz, E., et al. 2010, MNRAS, 404, 963

Kouveliotou, C., Meegan, C. A., Fishman, G. J., et al. 1993, ApJ, 413, L101

Lee, W. H., \& Ramirez-Ruiz, E. 2007, New J. Phys., 9, 17

Levan, A. J., Wynn, G. A., Chapman, R., et al. 2006, MNRAS, 368, L1

Levesque, E. M., Bloom, J. S., Butler, N. R., et al. 2010, MNRAS, 401, 963

Liang, E.-W., Zhang, B.-B., \& Zhang, B. 2007, ApJ, 670, 565

Mangano, V., \& Sakamoto, T. 2011, GCN, 12468, 1
Margutti, R., Genet, F., Granot, J., et al. 2010, MNRAS, 402, 46 Margutti, R., Zaninoni, E., Bernardini, M. G., et al. 2012, arXiv:1207.0537 Metzger, B. D., \& Berger, E. 2012, ApJ, 746, 48

Metzger, B. D., Quataert, E., \& Thompson, T. A. 2008, MNRAS, 385, 1455

Mochkovitch, R., Hernanz, M., Isern, J., \& Martin, X. 1993, Nature, 361, 236

Nakar, E., Gal-Yam, A., \& Fox, D. B. 2006, ApJ, 650, 281

Nakar, E., \& Granot, J. 2007, MNRAS, 380, 1744

Narayan, R., Paczynski, B., \& Piran, T. 1992, ApJ, 395, L83

Nicuesa Guelbenzu, A., Klose, S., Krühler, T., et al. 2012, A\&A, 538, L7

Nicuesa Guelbenzu, A., Klose, S., Rossi, A., et al. 2011, A\&A, 531, L6

Nousek, J. A., Kouveliotou, C., Grupe, D., et al. 2006, ApJ, 642, 389

Oates, S. R., \& Sakamoto, T. 2011, GCN, 12466, 1

Osborne, J. P., Beardmore, A. P., Evans, P. A., \& Goad, M. R. 2011, GCN, 12463,1

Panaitescu, A. 2006, MNRAS, 367, L42

Panaitescu, A., \& Kumar, P. 2001, ApJ, 560, L49

Perley, D. A., Cenko, S. B., Bloom, J. S., et al. 2009a, AJ, 138, 1690

Perley, D. A., Metzger, B. D., Granot, J., et al. 2009b, ApJ, 696, 1871

Perley, R. A., Chandler, C. J., Butler, B. J., \& Wrobel, J. M. 2011, ApJ, 739, L1

Perna, R., \& Belczynski, K. 2002, ApJ, 570, 252

Poole, T. S., Breeveld, A. A., Page, M. J., et al. 2008, MNRAS, 383, 627

Popham, R., Woosley, S. E., \& Fryer, C. 1999, ApJ, 518, 356

Predehl, P., \& Schmitt, J. H. M. M. 1995, A\&A, 293, 889

Qin, B., Wu, X.-P., Chu, M.-C., Fang, L.-Z., \& Hu, J.-Y. 1998, ApJ, 494, L57

Racusin, J. L., Liang, E. W., Burrows, D. N., et al. 2009, ApJ, 698, 43

Reddy, N. A., \& Steidel, C. C. 2009, ApJ, 692, 778

Rees, M. J., \& Meszaros, P. 1998, ApJ, 496, L1

Rezzolla, L., Giacomazzo, B., Baiotti, L., et al. 2011, ApJ, 732, L6

Rhoads, J. E. 1999, ApJ, 525, 737

Rosswog, S. 2005, Nuovo Cimento C Geophys. Space Phys. C, 28, 607

Rosswog, S., Ramirez-Ruiz, E., \& Davies, M. B. 2003, MNRAS, 345, 1077

Ruffert, M., \& Janka, H. 1999a, Prog. Theor. Phys. Suppl., 136, 287

Ruffert, M., \& Janka, H.-T. 1999b, A\&A, 344, 573

Sakamoto, T., Barthelmy, S. D., Baumgartner, W. H., et al. 2011b, GCN, 12464, 1

Sakamoto, T., Barthelmy, S. D., \& Norris, J. 2011a, GCN, 12477, 1

Sakamoto, T., Chester, M. M., Cummings, J. R., et al. 2011c, GCN, 12460, 1

Salvaterra, R., Cerutti, A., Chincarini, G., et al. 2008, MNRAS, 388, L6

Salvaterra, R., Devecchi, B., Colpi, M., \& D’Avanzo, P. 2010, MNRAS, 406, 1248

Sari, R., \& Mészáros, P. 2000, ApJ, 535, L33

Sari, R., Piran, T., \& Halpern, J. P. 1999, ApJ, 519, L17

Sari, R., Piran, T., \& Narayan, R. 1998, ApJ, 497, L17

Schlafly, E. F., \& Finkbeiner, D. P. 2011, ApJ, 737, 103

Schlegel, D. J., Finkbeiner, D. P., \& Davis, M. 1998, ApJ, 500, 525

Soderberg, A. M., Berger, E., Kasliwal, M., et al. 2006, ApJ, 650, 261

Stratta, G., D'Avanzo, P., Piranomonte, S., et al. 2007, A\&A, 474, 827

van der Horst, A. J., Kouveliotou, C., Gehrels, N., et al. 2009, ApJ, 699, 1087

Wakker, B. P., Lockman, F. J., \& Brown, J. M. 2011, ApJ, 728, 159

Watson, D. 2011, A\&A, 533, A16

Watson, D., Hjorth, J., Jakobsson, P., et al. 2006, A\&A, 454, L123

Willmer, C. N. A., Faber, S. M., Koo, D. C., et al. 2006, ApJ, 647, 853

Xin, L.-P., Liang, E.-W., Wei, J.-Y., et al. 2011, MNRAS, 410, 27

Zhang, B., Fan, Y. Z., Dyks, J., et al. 2006, ApJ, 642, 354

Zhang, B., \& Mészáros, P. 2002, ApJ, 566, 712 